\title{
Syntheses of methylated catechins and theaflavins using 2-nitrobenzenesulfonyl group to protect and deactivate phenol
}

\author{
Tomohiro Asakawa ${ }^{1}$, Yusuke Kawabe ${ }^{1}$, Atsushi Yoshida ${ }^{1}$, Yoshiyuki Aihara ${ }^{1}$, Tamiko Manabe ${ }^{1}$, \\ Yoshitsugu Hirose ${ }^{1}$, Asuka Sakurada ${ }^{1}$, Makoto Inai ${ }^{1}$, Yoshitaka Hamashima ${ }^{1}$, Takumi Furuta ${ }^{1,2}$, \\ Toshiyuki Wakimoto ${ }^{1,3}$ and Toshiyuki Kan $^{1}$
}

An efficient and versatile synthetic method for labile polyphenols was established using 2-nitrobenzenesulfonate (Ns) as a protecting group for phenol. This methodology provides regio- and stereoselective access to a range of methylated catechins, such as methylated epigallocatechin gallates, that are not readily available from natural sources. In addition, biomimetic synthesis of theaflavins from catechins was accomplished using Ns protection to minimize undesired side reactions of electron-rich aromatic rings during oxidation, enabling construction of the complex benzotropolone core in a single-step oxidative coupling reaction. Availability of these compounds will aid detailed structure-biological activity relationship studies of catechins. The Journal of Antibiotics (2016) 69, 299-312; doi:10.1038/ja.2016.14; published online 24 February 2016

\section{INTRODUCTION}

Synthetic methods for polyphenols have been extensively studied, because these compounds possess an array of interesting biological activities, including anticancer, antiviral and antimicrobial activities. Nevertheless, only a few efficient and versatile synthetic methods are available, both because the highly polar nature of polyphenols makes handling difficult, and because of the susceptibility of the compounds to oxidation. Protection of the hydroxyl groups of polyphenol is indispensable during usual chemical transformations and also during purification. Thus, selection of a suitable protecting group is critical. In this context, the 2-nitrobenzenesulfonate $(\mathrm{Ns})$ group $^{1}$ should be an excellent protecting group for labile polyphenols, because it can be easily removed under mild conditions. Furthermore, its electronwithdrawing nature should enhance the stability of polyphenols during synthetic manipulations. Herein, we report efficient syntheses of a range of methylated catechins ( 2 and 3 ) and theaflavins ( 4 and 5 ) by exploiting the Ns group for protection of phenols.

\section{RESULT AND DISCUSSION}

Synthesis of methylated cathechins

Epigallocatechin gallate (EGCg: 1), which has various biological activities, including cancer-preventive, antiviral and antimicrobial activities, is a major component of catechin derivatives derived from tea (Figure 1). ${ }^{2-4}$ Recently, various methylated derivatives, such as (-)-3'-methyl-epigallocatechin gallate (3'-Me-EGCg: 2), have been identified as minor catechins in natural tea leaves and as mammalian metabolites of tea catechins. ${ }^{4}$ Because 2 and its regioisomer
(4'-Me-EGCg: 3) exhibit potent inhibitory activities towards type I allergic reactions in mice ${ }^{5,6}$ and matrix metalloproteinases, ${ }^{7,8}$ it seems likely that other methylated catechin derivatives may also have therapeutic potential. ${ }^{9-11}$

Compounds $\mathbf{2}$ and $\mathbf{3}$ are readily available from natural and synthetic sources, ${ }^{12,13}$ and investigations of structure-activity relationships have so far been limited to these compounds. However, synthesis of all possible regio- and stereoisomers of the naturally available methylated catechins (EGC: epigallocatechin, GC: gallocatechin, EC: epicatechin and CC: catechin) would allow systematic evaluation of the structurebiological activity relationships. Hence, we are interested in concise synthesis of methylated catechins (2, 3 and 6-13). Although synthetic investigations of the catechin skeleton have been reported by many groups, ${ }^{2}$ including ours, ${ }^{14,15}$ derivation of methylated catechins from natural catechins, such as $\mathrm{EGC}^{16}$ and other derivatives (GC, EC and CC), which are inexpensive and readily available, should be a convenient and effective strategy.

Our synthetic plan is illustrated in Scheme 1. A key issue is selecting a suitable protecting group for phenol. Although benzyl ether has been employed for catechin synthesis because it is readily deprotectable under hydrogenolysis conditions, ether formation of phenol is often troublesome: for example, epimerization of the 2-position can occur under basic conditions (Scheme 2). To make matters worse, electronrich aromatics readily undergo undesired electrophilic substitution and unexpected oxidation at the benzylic position. Thus, a major concern in polyphenol synthesis is how to avoid these problems.

${ }^{1}$ School of Pharmaceutical Sciences, University of Shizuoka, Shizuoka, Japan; ${ }^{2}$ Institute for Chemical Research, Kyoto University, Kyoto, Japan and ${ }^{3}$ Faculty of Pharmaceutical Sciences, Hokkaido University, Hokkaido, Japan

Correspondence: Professor T Kan, School of Pharmaceutical Sciences, University of Shizuoka, 52-1 Yada, Suruga-ku, Shizuoka 422-8526, Japan.

E-mail: kant@u-shizuoka-ken.ac.jp

Received 8 December 2015; revised 26 January 2016; accepted 26 January 2016; published online 24 February 2016 
<smiles>[R]c1cc(C(=O)O[C@@H]2Cc3c(O)cc(O)cc3OC2c2cc([R])c([R])c(O)c2)cc(O)c1[R7]</smiles>

\begin{tabular}{|c|c|c|c|}
\hline catechines & & $\mathrm{R}^{1} \quad \mathrm{R}^{2}$ & $\mathrm{R}^{3}$ \\
\hline$(-)-E G C g(1):$ & 2,3-cis & $\mathrm{OH} \mathrm{OH}$ & $\mathrm{OH} \mathrm{OH}$ \\
\hline (-)-3"-Me-EGCg (2): & $2,3-c i s$ & $\mathrm{OH} \mathrm{OH}$ & $\mathrm{OMeOH}$ \\
\hline$(-)-4 "-M e-E G C g(3):$ & 2,3-cis & $\mathrm{OH} \mathrm{OH}$ & $\mathrm{OH} \mathrm{OMe}$ \\
\hline$(-)-3^{\prime}-M e-E G C g(6):$ & 2,3-cis & $\mathrm{OH} \mathrm{OM}$ & $\mathrm{eOH} \mathrm{OH}$ \\
\hline (-)-4'-Me-EGCg (7): & $2,3-$ cis & $\mathrm{OMeOH}$ & $\mathrm{OH} \mathrm{OH}$ \\
\hline$(-)-3^{\prime \prime}-\mathrm{Me}-\mathrm{ECg}(8)$ : & $2,3-c i s$ & $\mathrm{OH} \mathrm{H}$ & $\mathrm{OMeOH}$ \\
\hline$(-)-4 "-M e-E C g(9):$ & 2,3-cis & $\mathrm{OH} \mathrm{H}$ & $\mathrm{OH} \mathrm{OMe}$ \\
\hline (-)-3"-Me-GCg (10): & 2,3-trans & $\mathrm{OH} \mathrm{OH}$ & $\mathrm{OMeOH}$ \\
\hline (-)-4"-Me-GCg (11): & 2,3-trans & $\mathrm{OH} \mathrm{OH}$ & $\mathrm{OH} \mathrm{OMe}$ \\
\hline (+)-3"-Me-CCg (12): & 2,3-trans & $\mathrm{OH} \mathrm{H}$ & $\mathrm{OMeOH}$ \\
\hline$(+)-4 "-M e-C C g(13):$ & 2,3-trans & $\mathrm{OH} \mathrm{H}$ & $\mathrm{OH} \mathrm{OMe}$ \\
\hline
\end{tabular}

Figure 1 EGCg (1) and methylated catechins.

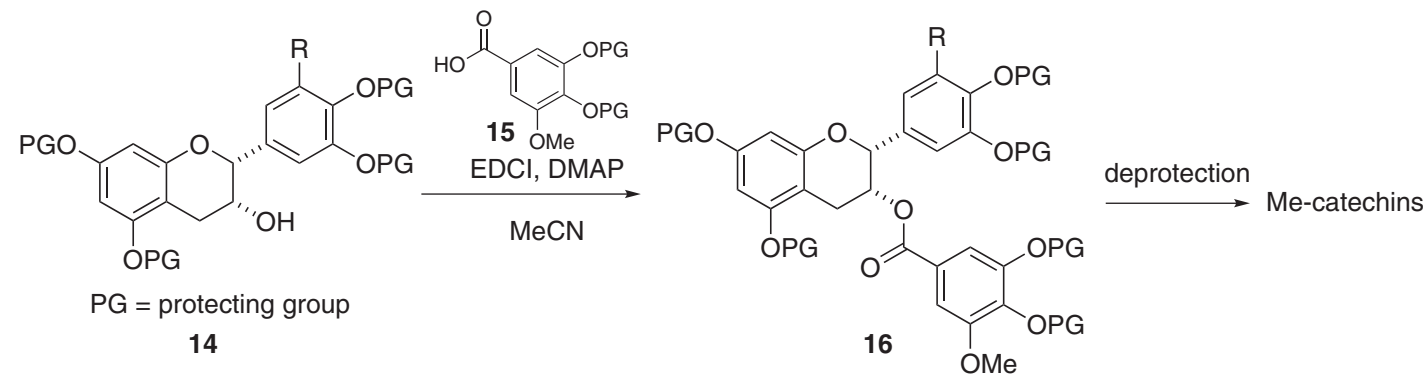

Scheme 1 Plan for practical synthesis of methylated catechins.

-Epimerization via quinonmethide

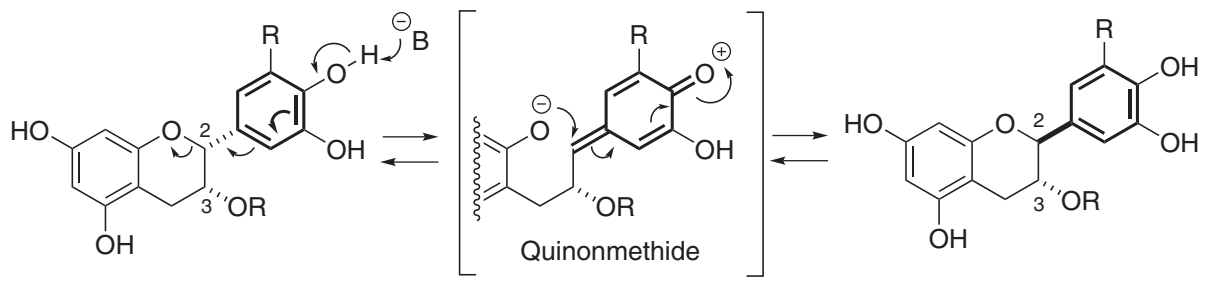

.Electrophilic substitution and oxidation

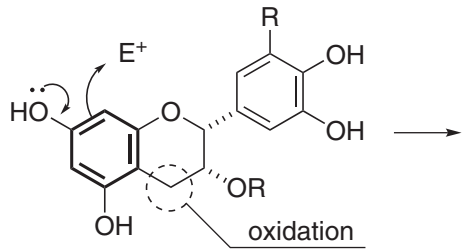<smiles>[R]c1cc([C@@H]2Oc3c(F)c(O)cc(O)c3C(=O)C2(O)O)cc(O)c1O</smiles>

Scheme 2 Potential issues in catechin synthesis.

The Ns group should be an excellent protecting group for labile polyphenols because it can be easily deprotected under mild conditions. ${ }^{1,17-19}$ Furthermore, the electron-withdrawing nature of the Ns group should improve the stability of polyphenol during synthetic manipulations. A preliminary investigation of deprotection of 4-(((2-nitrophenyl)sulfonyl)oxy)benzyl acetate (17) showed that selective deprotection of the Ns group could be achieved in the presence of the ester group (Scheme 3). Thus, protection of phenols with Ns group should be effective for the synthesis of methylated catechins.

Therefore, we next investigated selective incorporation of the methyl group at 3- and 4-OH of allyl gallate (20), as shown in Scheme 4. Gallic acid (19) was converted to allyl ester 20 by reaction with allyl alcohol and EDCI. Upon treatment of 20 with $\mathrm{Li}_{2} \mathrm{CO}_{3}$ and methyl iodide, selective deprotonation and alkylation of the most acidic 4-OH proceeded smoothly to give 21 . On the other hand, 3-OH selective alkylation was achieved by utilizing a bridged boronic ester intermediate $^{20}$ between the $o$-phenolic hydroxyl groups. After formation of the boronic ester $\mathbf{2 3}$ by treating $\mathbf{2 0}$ with borax in the presence of $\mathrm{NaOH}$, methylation with $\mathrm{Me}_{2} \mathrm{SO}_{4}$ and subsequent acidic hydrolysis of the boronic ester exclusively provided 24 . Ns groups were incorporated into the resultant phenols 20,21 and 24 by treatment with $\mathrm{NsCl}$ and $\mathrm{Et}_{3} \mathrm{~N}$. Treatment of the allyl esters with catalytic amounts of $\mathrm{Pd}\left(\mathrm{PPh}_{3}\right)_{4}$ and $p$-tolSO $\mathrm{Na}^{21}$ resulted in smooth deprotection of the allyl group to afford the desired 26, 22 and 25, respectively. 
Next, we focused on incorporating gallate derivatives $\mathbf{2 2}$ and $\mathbf{2 5}$ into protected epigallocatechin derivatives and deprotection of the Ns group (Scheme 5). Protection of EGC (27) with the Ns group was carried out with $\mathrm{NsCl}$ and $\mathrm{Et}_{3} \mathrm{~N}$ to provide 28. Condensation reaction of 28 with 25 and EDCI in the presence of a catalytic amount of DMAP proceeded smoothly to provide the desired 29 in high yield. Similar condensation of $\mathbf{2 8}$ and $\mathbf{2 2}$ provided the $4^{\prime}$-methylated EGCg derivative 30. Deprotection of the Ns groups of 29 and 30 was accomplished with the use of thiophenol and cesium carbonate to provide 3'-methylated EGCg (2) and 4'-methylated EGCg (3), respectively. During this transformation, neither epimerization at the 2-position of the benzopyran ring nor decomposition of gallate ester was observed. A similar protocol provided the desired methylated gallate catechin derivatives $\mathbf{8}-\mathbf{1 3}$ from natural catechin derivatives GC, EC and CC (Table 1). ${ }^{22}$

Then, we turned our attention to selectively introducing a methyl group into the B-ring (Scheme 6). To our knowledge, (-)-EGCg derivatives methylated at the B-ring have not yet been reported, so an SAR study of these compounds should be interesting. We found that a bridged boronic ester intermediate effectively distinguishes the hydroxyl groups at the A-ring and B-ring. Treatment of 27 with
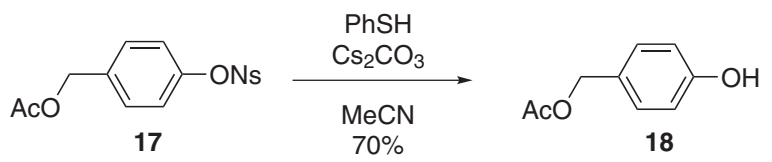

Scheme 3 Selective deprotection of $\mathrm{O}$-Ns group. three equivalents of $\mathrm{NsCl}$ and excess $\mathrm{H}_{3} \mathrm{BO}_{3}$ in the presence of $\mathrm{NaOH}^{23}$ resulted in regioselective sulfonylation to afford predominantly 3',5,7-Ns-EGCg (32).

Next, selective incorporation of the TBDPS group at the less hindered hydroxyl group $\left(5^{\prime}-\mathrm{OH}\right)$ was accomplished by treating 32 with TBDPSCl and $\mathrm{Et}_{3} \mathrm{~N}$ to give 33 (Scheme 7). The 4'-Me-EGCg derivative was prepared by methylation of 33 with diazomethane, condensation of 35 with Ns-protected gallic acid 26, ${ }^{24}$ and stepwise deprotection of TBDPS group and Ns group, affording 4'-Me-EGCg (7).

3'-Me-EGCg (6) and 3',3'-diMe-EGCg (38) were also synthesized from 33. Protection of $\mathbf{3 3}$ with Ns proceeded at the $4^{\prime}$-hydroxyl group. Deprotection of the TBDPS group and incorporation of a methyl group afforded 37. Although condensation of 37 with 26 and deprotection of Ns groups readily provided 3'-Me-EGCg (6), we chose to employ a modified preparation with double-methylated derivatives. After condensation of 37 and 25, deprotection of Ns groups was accomplished using 2-aminothiophenol (39) instead of thiophenol to afford 3', $3^{\prime}$-diMe- EGCG (38; Table 1). ${ }^{25,26}$ For a review on recent progress in the synthesis of the advantage of this method is that 39 is odorless compared with thiophenol. Furthermore, the by-product, 2-(2-nitrophenylthio)aniline (40), can be easily removed by washing the ethereal layer with $1 \mathrm{M} \mathrm{HCl}$ solution.

Combining the selective methylation strategies for the B- and D-rings of EGCg should provide access to several types of doublemethylated EGCg derivatives. Furthermore, this regioselective modification of EGCg should be applicable for alkylation as well as acylation; thus, it should be possible to apply this synthetic strategy to<smiles>O=C(O)c1cc(O)c(O)c(Br)c1</smiles>

19

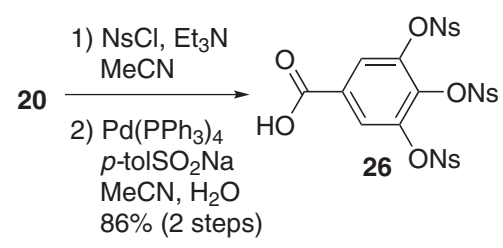

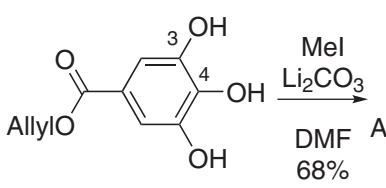

20<smiles>OC1CC1</smiles><smiles>COC(=O)c1cc(O)c(O)c(O)c1</smiles>
21

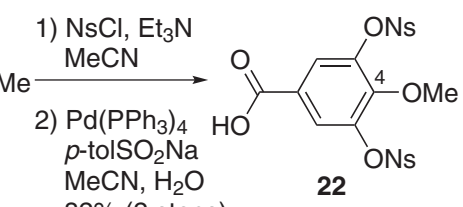

$82 \%$ (2 steps)

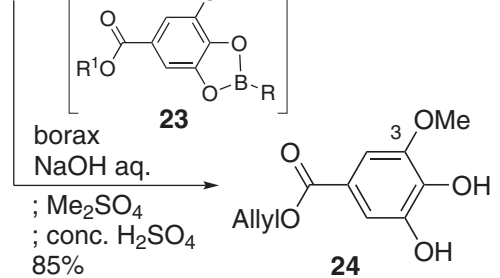

$85 \%$

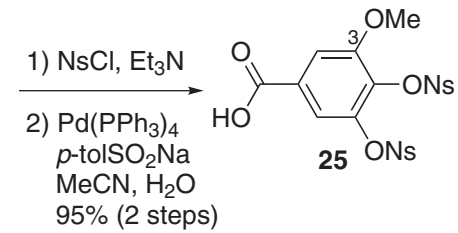

Scheme 4 Synthesis of selectively methylated gallic acids.<smiles>[R6]c1cc([2H])c2c(c1)O[C@H](c1cc(O)c([R])c([18OH])c1)[C@H](O)C2</smiles>

Scheme 5 Synthesis of 3'-Me-EGCg (2) and 4'-Me-EGCg (3). 


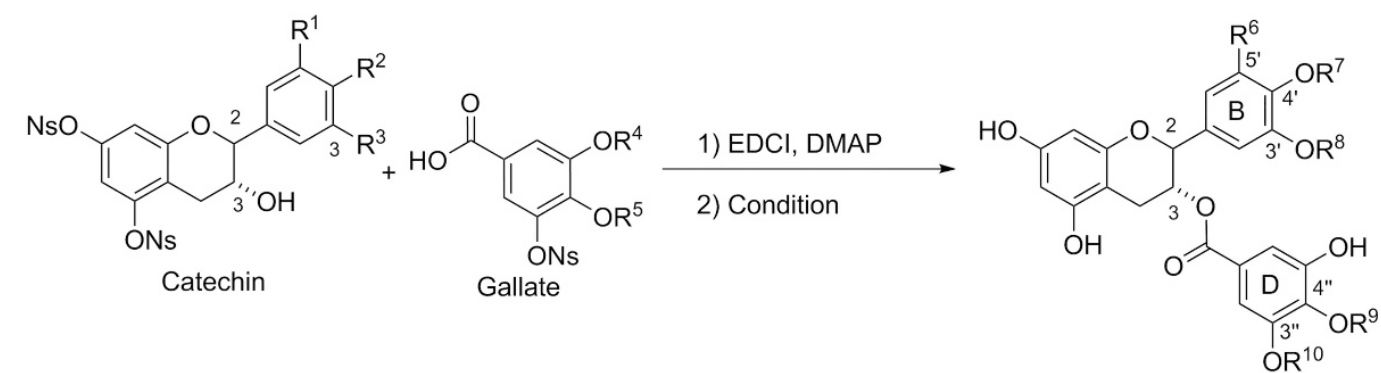

\begin{tabular}{|c|c|c|c|c|c|}
\hline Entry & Catechin & Gallate & Condition & Product & Yield(\%) \\
\hline 1 & $\begin{aligned} 35: \mathrm{R}^{1} & =\mathrm{ONs}, \mathrm{R}^{2}=\mathrm{OMe} \\
\mathrm{R}^{3} & =\text { OTBDPS, } 2,3 \text {-cis }\end{aligned}$ & $26: R^{4,5}=N s$ & $\begin{array}{l}\text { 1) TBAF, AcOH } \\
\text { THF } \\
\text { 2) Conditon A }\end{array}$ & $\begin{array}{r}\text { 4'-Me-EGCg (7) }:^{\mathrm{R}^{6}=\mathrm{OH}, \mathrm{R}^{7}=\mathrm{Me}} \\
\mathrm{R}^{8,9,10}=\mathrm{H}, 2,3 \text {-cis }\end{array}$ & 70 \\
\hline 2 & $\begin{aligned} 37: & \mathrm{R}^{1}=\mathrm{OMe} \\
& \mathrm{R}^{2,3}=\mathrm{ONs}, 2,3-\mathrm{cis}\end{aligned}$ & $26: R^{4,5}=N s$ & Condition A & $\begin{aligned} \text { 3'-Me-EGCg (6) : } & \mathrm{R}^{6}=\mathrm{OH}, \mathrm{R}^{8}=\mathrm{Me} \\
& \mathrm{R}^{7,9,10}=\mathrm{H}, 2,3-\mathrm{cis}\end{aligned}$ & 63 \\
\hline 3 & $\begin{aligned} 37: & \mathrm{R}^{1}=\mathrm{OMe} \\
& \mathrm{R}^{2,3}=\mathrm{ONs}, 2,3-\mathrm{cis}\end{aligned}$ & $\begin{aligned} 25: R^{4} & =M e \\
R^{5} & =N s\end{aligned}$ & Condition B & $\begin{array}{c}\text { 3',3"-diMe-EGCg (38) : } \mathrm{R}^{6}=\mathrm{OH}, \mathrm{R}^{8,10}=\mathrm{Me} \\
\mathrm{R}^{7,9}=\mathrm{H}, 2,3-\text { cis }\end{array}$ & 52 \\
\hline 4 & $\begin{array}{l}41: \mathrm{R}^{1}=\mathrm{H}, \mathrm{R}^{2,3}=\mathrm{ONs} \\
\quad 2,3 \text {-cis }\end{array}$ & $\begin{aligned} 25: \mathrm{R}^{4} & =\mathrm{Me} \\
\mathrm{R}^{5} & =\mathrm{Ns}\end{aligned}$ & Condition A & $\begin{aligned} \text { 3"-Me-ECg (8) }: & R^{6}=H, R^{7,8,9}=H \\
& R^{10}=M e, 2,3-c i s\end{aligned}$ & 84 \\
\hline 5 & $\begin{array}{l}41: \mathrm{R}^{1}=\mathrm{H}, \mathrm{R}^{2,3}=\mathrm{ONs} \\
\quad 2,3 \text {-cis }\end{array}$ & $\begin{aligned} 22: R^{4} & =N s \\
R^{5} & =M e\end{aligned}$ & Condition A & $\begin{aligned} \text { 4"-Me-ECg (9) : } & \mathrm{R}^{6}=\mathrm{H}, \mathrm{R}^{7,8,10}=\mathrm{H} \\
\mathrm{R}^{9} & =\mathrm{Me}, 2,3-\text { cis }\end{aligned}$ & 83 \\
\hline 6 & $\begin{array}{l}\text { 42: } \mathrm{R}^{1}=\mathrm{OH}, \mathrm{R}^{2,3}=\mathrm{ONs} \\
\text { 2,3-trans }\end{array}$ & $\begin{aligned} 25: \mathrm{R}^{4} & =\mathrm{Me} \\
\mathrm{R}^{5} & =\mathrm{Ns}\end{aligned}$ & Condition A & $\begin{aligned} \text { 3"-Me-GCg (10): } & \mathrm{R}^{6}=\mathrm{OH}, \mathrm{R}^{7,8,9}=\mathrm{H} \\
& \mathrm{R}^{10}=\mathrm{Me}, 2,3 \text {-trans }\end{aligned}$ & 75 \\
\hline 7 & $\begin{array}{l}\text { 42: } \mathrm{R}^{1}=\mathrm{OH}, \mathrm{R}^{2,3}=\mathrm{ONs} \\
\text { 2,3-trans }\end{array}$ & $\begin{aligned} 22: \mathrm{R}^{4} & =\mathrm{Ns} \\
\mathrm{R}^{5} & =\mathrm{Me}\end{aligned}$ & Condition A & $\begin{aligned} \text { 4"-Me-GCg (11) : } & \mathrm{R}^{6}=\mathrm{OH}, \mathrm{R}^{7,8,10}=\mathrm{H} \\
\mathrm{R}^{9} & =\mathrm{Me}, 2,3 \text {-trans }\end{aligned}$ & 71 \\
\hline 8 & $\begin{aligned} 43: & \mathrm{R}^{1}=\mathrm{H}, \mathrm{R}^{2,3}=\mathrm{ONs} \\
& \text { 2,3-trans }\end{aligned}$ & $\begin{aligned} 25: R^{4} & =M e \\
R^{5} & =N s\end{aligned}$ & Condition A & $\begin{aligned} \text { 3"-Me-CCg (12) : } & R^{6}=H, R^{7,8,9}=H \\
& R^{10}=M e, 2,3 \text {-trans }\end{aligned}$ & 73 \\
\hline 9 & $\begin{array}{l}43: \mathrm{R}^{1}=\mathrm{H}, \mathrm{R}^{2,3}=\mathrm{ONs} \\
\text { 2,3-trans }\end{array}$ & $\begin{aligned} 22: R^{4} & =\mathrm{Ns} \\
\mathrm{R}^{5} & =\mathrm{Me}\end{aligned}$ & Condition A & 4"-Me-CCg (13): $\begin{aligned} \mathrm{R}^{6} & =\mathrm{H}, \mathrm{R}^{7,8,10}=\mathrm{H} \\
\mathrm{R}^{9} & =\mathrm{Me}, 2,3 \text {-trans }\end{aligned}$ & 79 \\
\hline
\end{tabular}

\begin{tabular}{|c|c|}
\hline $\begin{array}{c}\text { Condition A } \\
\text { PhSH } \\
\mathrm{Cs}_{2} \mathrm{CO}_{3} \\
\mathrm{MeCN}\end{array}$ & $\mathrm{Cs}_{2} \mathrm{CO}_{3}, \mathrm{MeCN}$ \\
\hline
\end{tabular}<smiles>Oc1cc(O)c2c(c1)O[C@H](c1cc(O)c(O)c(O)c1)[C@H](O)C2</smiles>

Scheme 6 Selective incorporation of Ns group via bridged boronic ester intermediate.

other natural catechins (GC, EC and C) to construct a diverse catechin library.

\section{Synthesis of theaflavins}

Theaflavin (4) is an oxidative dimer of catechin derivatives (Figure 2), ${ }^{27-30}$ which is found in black tea. It has a number of biological activities. ${ }^{31}$ Although many synthetic studies of catechin derivatives have been reported, ${ }^{32-34}$ there are only a few reports of the synthesis of $\mathbf{4}$ by means of enzymatic oxidation. ${ }^{32,35}$
Recently, Nakatsuka and Yanase's group reported a novel oxidative coupling of $o$-quinone 45 and pyrogallol derivative 46 based on their proposed biosynthetic pathway, ${ }^{35}$ as shown in Scheme 8. Although their model reaction proceeded in excellent yield, its application to the synthesis of theaflavin has not been reported to date. This is probably because oxidation precursors and theaflavin itself are unstable under strongly oxidizing conditions. We envisioned that the application of our Ns group ${ }^{16-19}$ for protecting reactive phenols would enable efficient synthesis of theaflavins from catechins. 
<smiles>COc1cc(OC(C)(C)C)cc2c1C[C@@H](O)[C@@H](c1cc(O)c(O)c(O[Si](C)(C)C)c1)O2</smiles>

34

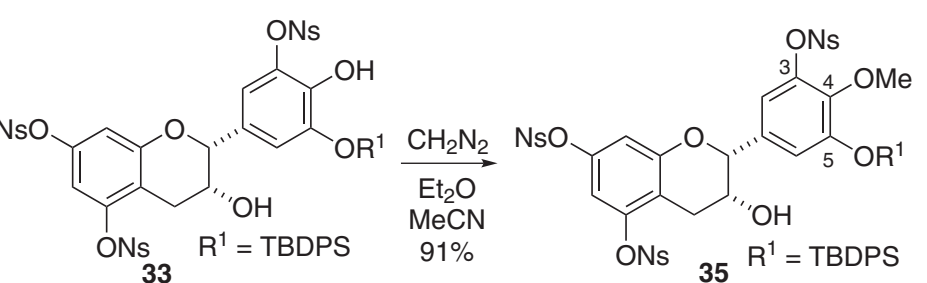

1) $\mathrm{NsCl} \mathrm{Et}{ }_{3} \mathrm{~N}, \mathrm{MeCN}, \mathrm{H}_{2} \mathrm{O}$

$95 \%$

2) $\mathrm{TBAF}, \mathrm{AcOH}, \mathrm{THF}$

$86 \%$<smiles>CCO[N+]([O-])(O)O[Na]</smiles>

Scheme 7 Synthesis of selectively methylated epigallocatechin.<smiles>O=c1c(O)cc(C2Oc3cc(O)cc(O)c3CC2O)cc2c([C@@H]3Oc4cc(O)cc(O)c4C[C@H]3O)cc(O)c(O)c12</smiles>

theaflavin (4): 2,3-cis

neotheaflavin (5): 2,3-trans

Figure 2 Structures of theaflavine (4) and neotheaflavine (5).

For the synthesis of theaflavin, we initially investigated selective incorporation of the Ns group into the A-ring of CC (49), EC (50) and EGC (27), as shown in Table 2.

Regioselective protection of the A-ring with a Ns group was performed utilizing the bridged boronic ester intermediate $54^{11}$ between neighboring phenolic hydroxyl groups on the B-ring (Scheme 9). Thus, after formation of the boronic ester by treatment of 27 with boronic acid in the presence of $\mathrm{NaOH}$, reaction with two equivalents of $\mathrm{NsCl}$ and subsequent acidic hydrolysis of boronic ester exclusively provided 53 (Table 2, entry 3 ). Although the boronic ester intermediate of $\mathbf{2 7}$ possesses a remaining phenolic hydroxyl group on the B-ring, steric hindrance appears to prevent reaction with $\mathrm{NsCl}$. Similar reactions of $\mathbf{4 9}$ and $\mathbf{5 0}$ proceeded smoothly to provide $\mathbf{5 1}$ and 52 (entries 1 and 2).

Having succeeded in selective protection of the A-ring, the next challenge is oxidation of the catechol group in $\mathbf{5 1}$ and $\mathbf{5 2}$. First, we optimized the oxidation conditions using 51, which is derived from less expensive 49. As shown in Scheme 10, the best result was obtained by oxidation with $\mathrm{Pb}(\mathrm{OAc})_{4}$ in $\mathrm{MeCN}$ at $0{ }^{\circ} \mathrm{C}$, affording the desired quinone 55 in good yield. Utilizing $\mathrm{Pb}(\mathrm{OAc})_{4}$ as an oxidant has the advantage that lead derivatives can be easily removed from the reaction medium by celite filtration after completion of the reaction. Furthermore, the electron-withdrawing nature of the Ns group would
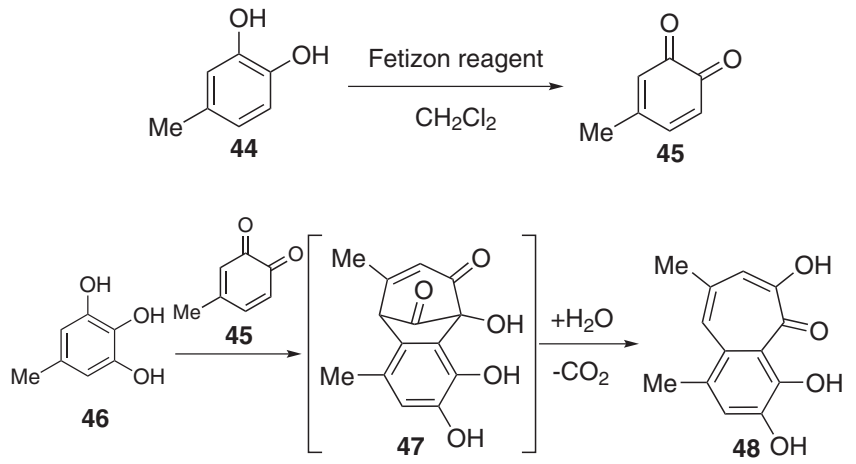

Scheme 8 Coupling reaction of o-quinone $\mathbf{4 5}$ and pyrogallol derivative $\mathbf{4 6}$ reported by Nakatsuka and Yanase.

enhance the stability of the A-ring during the reaction with the strong oxidant $\mathrm{Pb}(\mathrm{OAc})_{4}$. Although other various oxidants (such as Fetizon reagent and $\mathrm{PhI}(\mathrm{OAc})_{2}$ ) were tested, they did not efficiently provide the $o$-quinone for the next reaction without the need for purification.

Next, we tested the coupling reaction of quinone $\mathbf{5 5}$ with pyrogallol derivative 53 (Scheme 11). As quinone 55 was labile during purification, crude 55 was used directly for the coupling reaction. Gratifyingly, treatment of three equivalents of $\mathbf{5 5}$ with $\mathbf{5 3}$ in a mixture of $\mathrm{MeCN}$ and $\mathrm{CH}_{2} \mathrm{Cl}_{2}$, followed by addition of $\mathrm{H}_{2} \mathrm{O}$, provided the desired 56 in moderate yield. Considering the reaction mechanism shown in Scheme 12, at least two equivalents of o-quinone should theoretically be consumed in the two oxidation steps (58-59 and 61-62). Furthermore, addition of MS3A to the reaction mixture before the addition of $\mathrm{H}_{2} \mathrm{O}$ was essential for efficient conversion. MS3A might have a significant role in the oxidation step from $\mathbf{5 8}$ to 59, although the mechanism involved remains to be established. After separation from 51 (generated by reduction of 55), deprotection of the Ns group of $\mathbf{5 6}$ was accomplished by treatment with thiophenol and cesium carbonate to provide neotheaflavin (5). No decomposition of the reactive benzotropolone ring was observed during the coupling and deprotection processes. 


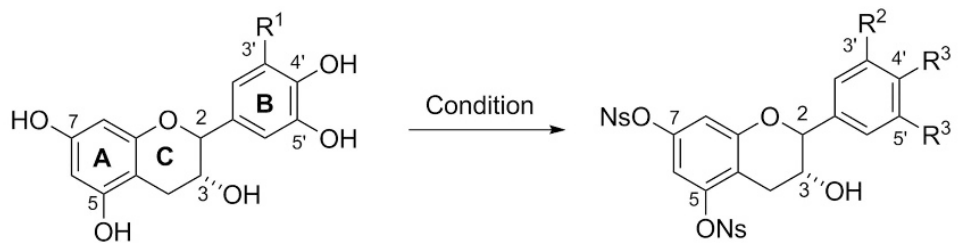

\begin{tabular}{|c|c|c|c|c|}
\hline Entry & Catechin & Condition & Product & Yield(\%) \\
\hline 1 & $49: \mathrm{R}^{1}=\mathrm{H}, 2,3$-trans & $\begin{array}{l}\text { exess } \mathrm{B}(\mathrm{OH})_{3} \\
\mathrm{NaOH} \text { aq. }(\mathrm{pH} 9.0) \\
\mathrm{NsCl}(2 \text { eq), toluene }\end{array}$ & 5,7-Ns-CC (51): $\mathrm{R}^{2}=\mathrm{H}, \mathrm{R}^{3}=\mathrm{OH}$ & 70 \\
\hline 2 & $50: \mathrm{R}^{1}=\mathrm{H}, 2,3-c i s$ & $\begin{array}{l}\text { exess } \mathrm{B}(\mathrm{OH})_{3} \\
\mathrm{NaOH} \text { aq. }(\mathrm{pH} 9.0) \\
\mathrm{NsCl}(2 \text { eq), toluene }\end{array}$ & $5,7-\mathrm{Ns}-\mathrm{EC}(\mathbf{5 2}): \mathrm{R}^{2}=\mathrm{H}, \mathrm{R}^{3}=\mathrm{OH}$ & 63 \\
\hline 3 & $27: \mathrm{R}^{1}=\mathrm{OH}, 2,3$-cis & $\begin{array}{l}\text { exess } \mathrm{B}(\mathrm{OH})_{3} \\
\mathrm{NaOH} \text { aq. }(\mathrm{pH} 9.0) \\
\mathrm{NsCl}(2 \text { eq), toluene }\end{array}$ & $5,7-\mathrm{Ns}-\mathrm{EGC}(\mathbf{5 3}): \mathrm{R}^{2}, \mathrm{R}^{3}=\mathrm{OH}$ & 52 \\
\hline 4 & $27: \mathrm{R}^{1}=\mathrm{OH}, 2,3$-cis & $\begin{array}{l}\text { exess } \mathrm{B}(\mathrm{OH})_{3} \\
\mathrm{NaOH} \text { aq. }(\mathrm{pH} 9.0) \\
\mathrm{NsCl}(3 \mathrm{eq}), \text { toluene }\end{array}$ & $5,7,3^{\prime}-N s-E G C(32): R^{2}=O N s, R^{3}=O H$ & 40 \\
\hline 5 & $27: \mathrm{R}^{1}=\mathrm{OH}, 2,3$-cis & $\mathrm{NsCl}, \mathrm{Et}_{3} \mathrm{~N}, \mathrm{MeCN},-20^{\circ} \mathrm{C}$ & $5,7,3^{\prime}, 4^{\prime}, 5^{\prime}-N s-E G C(28): R^{2}, R^{3}=$ ONs & 94 \\
\hline
\end{tabular}

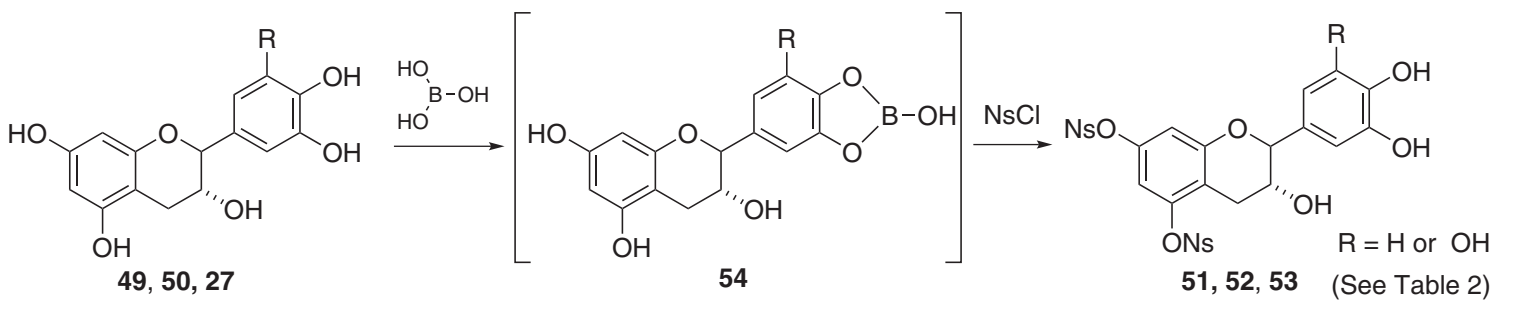

Scheme 9 Regioselective incorporation of Ns group into the A-ring.

This benzotropolone ring-forming reaction was also applicable to the synthesis of theaflavin (4) and its derivatives, as shown in Scheme 13. Upon treatment of Ns-protected epicatechin (52) with $\mathrm{Pb}(\mathrm{OAc})_{4}$, the desired oxidation reaction proceeded smoothly to provide the $o$-quinone $\mathbf{6 3}$. The coupling reaction with $\mathbf{5 3}$ was carried out without purification of the $o$-quinone intermediate 63 to give benzotropolone intermediate 64. Finally, deprotection of Ns group afforded theaflavin (4). ${ }^{36}$ The lower yield of $\mathbf{4}$ in comparison with 5 might be a result of instability of the $o$-quinone intermediate derived from cis-benzopyran 52.

This oxidation-coupling strategy was also applied to the coupling between pyrogallol (65) and gallate 66 to give 67 and 68 , respectively (Table 3). As compounds similar to $\mathbf{6 8}$, which is derived from oxidative coupling with gallate derivatives, have been isolated from tea, ${ }^{8}$ this method would be useful to synthesize derivatives for SAR study.

In summary, we have shown that the Ns group is an excellent protecting group for labile polyphenols; it can be easily deprotected under mild conditions, and its electron-withdrawing nature improves the stability of polyphenol during synthetic manipulations. Utilizing the Ns group to protect phenols and to block undesired oxidation reactions, we were able to develop an efficient and practical method for regio- and stereoselective methylation of catechins and theaflavins. Further applications, as well as structure-biological activity relationship studies of the synthesized derivatives, are under investigation in our laboratory.

\section{EXPERIMENTAL SECTION}

\section{General experimental details}

NMR $\left[{ }^{1} \mathrm{H}\right.$ NMR $(270 \mathrm{MHz}),{ }^{13} \mathrm{C}$ NMR $\left.(68 \mathrm{MHz})\right]$ spectra were determined on a JEOL EX-270 instrument (Tokyo, Japan), and $\left[{ }^{1} \mathrm{H}\right.$ NMR $(500 \mathrm{MHz}),{ }^{13} \mathrm{C}$ NMR (125 MHz)] spectra were determined on JEOL $\alpha-500$ instrument (Tokyo, Japan). Chemical shifts for ${ }^{1} \mathrm{H}$ NMR were reported in p.p.m. downfields from tetramethylsilane $(\delta)$ as the internal standard and coupling constants are in hertz $(\mathrm{Hz})$. The following abbreviations are used for spin multiplicity: $\mathrm{s}=$ singlet, $\mathrm{d}=$ doublet, $\mathrm{t}=$ triplet, $\mathrm{q}=$ quartet, $\mathrm{m}=$ multiplet, $\mathrm{br}=$ broad. Chemical shifts for ${ }^{13} \mathrm{C}$ NMR were referenced to solvent peaks: $\delta_{\mathrm{C}} 77.0$ for $\mathrm{CDCl}_{3}$, $\delta_{\mathrm{C}} 29.8$ for acetone- $d_{6}, \delta_{\mathrm{C}} 118.3$ for $\mathrm{CD}_{3} \mathrm{CN}$ and $\delta_{\mathrm{C}} 49.0$ for $\mathrm{CD}_{3} \mathrm{OD}$. HR-MS were obtained on either JEOL MStation JMS-700 or JMS-GCmate II (Tokyo, Japan). Fast atom bombardment (FAB) mass spectra were obtained with a mixture of 3-nitrobenzylalcohol and magic bullet as a matrix.

Analytical TLC was performed on Merck (Tokyo, Japan) precoated analytical plates, $0.25-\mathrm{mm}$ thick, silica gel $60 \mathrm{~F}_{254}$. Preparative TLC separations were made on $7 \times 20-\mathrm{cm}$ plates prepared with a $0.25-\mathrm{mm}$ layer of Merck silica gel $60 \mathrm{~F}_{254}$. Compounds were eluted from the adsorbent with $10 \%$ methanol in chloroform. Column chromatography was carried out with KANTO CHEMICAL (Tokyo, Japan) Silica Gel $60 \mathrm{~N}$ (spherical, neutral) 63-210 $\mu \mathrm{m}$. Reagents and solvents were commercial grades and were used as supplied with following exceptions: dichloromethane, diethylether, $n$-hexane, tetrahydrofuran and toluene, dried over molecular sieves 4A. All reactions sensitive to oxygen or moisture were conducted under an argon atmosphere.

\section{Experimental procedures and characterization data}

Allyl gallate (20). To a mixture of gallic acid monohydrate $(50.0 \mathrm{~g}$, $266 \mathrm{mmol}$ ), 1-(3-Dimethylaminopropyl)-3-ethylcarbodiimide hydrochloride 
(EDCI) $(61.2 \mathrm{~g}, 319 \mathrm{mmol})$ and 4-Dimethylaminopyridine (DMAP) $(3.25 \mathrm{~g}$, $26.6 \mathrm{mmol})$ was added allyl alcohol $(200 \mathrm{ml})$ at room temperature, then heated at $60^{\circ} \mathrm{C}$ for $4 \mathrm{~h}$. The resulting mixture was quenched with $2 \mathrm{~N} \mathrm{HCl}$ and extracted with ethyl acetate. The combined organic phases were washed with saturated $\mathrm{NaHCO}_{3}$, dried over anhydrous sodium sulfate and evaporated under reduced pressure. The residue was recrystallized from ethyl acetate to afford $\mathbf{2 0}$ $(48.7 \mathrm{~g}, 87 \%)$ as a light tan solid. HR-MS (FAB) calculated for $\mathrm{C}_{10} \mathrm{H}_{11} \mathrm{O}_{5}[\mathrm{M}$ $+\mathrm{H}]^{+} 211.0606$, found 211.0593; IR (neat) 3367, 1701, 1610, 1388, 1256, $1198 \mathrm{~cm}^{-1} ;{ }^{1} \mathrm{H}$ NMR $\left(270 \mathrm{MHz}\right.$, acetone- $\left.d_{6}\right) \delta 7.13(2 \mathrm{H}, \mathrm{s}), 5.97-6.11(1 \mathrm{H}, \mathrm{m})$, $5.37\left(1 \mathrm{H}, \mathrm{dq}, J_{1,2}=17.1,1.8 \mathrm{~Hz}\right), 5.22\left(1 \mathrm{H}, \mathrm{dq}, J_{1,2}=10.6,1.8 \mathrm{~Hz}\right), 4.71(2 \mathrm{H}$, $\left.\mathrm{dt}, J_{1,2}=5.5,1.8 \mathrm{~Hz}\right) ;{ }^{13} \mathrm{C}$ NMR $\left(68 \mathrm{MHz}\right.$, acetone- $\left.d_{6}\right) \delta 166.3,146.0,138.8$, $133.9,121.7,117.6,109.8,65.4$.

Allyl 3,5-dihydroxy-4-methoxybenzoate (21). To a suspension of $20(1.50 \mathrm{~g}$, $7.15 \mathrm{mmol})$ in $\mathrm{N}, \mathrm{N}$-dimethylformamide (DMF) $(20 \mathrm{ml})$ were added $\mathrm{Li}_{2} \mathrm{CO}_{3}$ $(1.32 \mathrm{~g}, 17.9 \mathrm{mmol})$ and methyl iodide $(1.11 \mathrm{ml}, 17.9 \mathrm{mmol})$ at $50^{\circ} \mathrm{C}$. The mixture was stirred at $50{ }^{\circ} \mathrm{C}$ for $20 \mathrm{~h}$. The reaction mixture was quenched with $2 \mathrm{M} \mathrm{HCl}$ and extracted with ethyl acetate. The combined organic phases were<smiles>COc1cc(O[Na])c2c(c1)O[C@H](C1=CC(=O)C(=O)C=C1)[C@H](O)C2</smiles>

Scheme 10 Oxidation of 51 to o-quinone 55 . washed with brine, dried over anhydrous sodium sulfate and evaporated under reduced pressure. The residue was purified by silica gel flash column chromatography $\left(1-3 \% \mathrm{MeOH}\right.$ in $\left.\mathrm{CH}_{2} \mathrm{Cl}_{2}\right)$ to afford $21(1.83 \mathrm{~g}, 68 \%)$ as a colorless solid. HR-MS (FAB) calculated for $\mathrm{C}_{11} \mathrm{H}_{12} \mathrm{O}_{5}[\mathrm{M}]^{+} 224.0685$, found 224.0705; IR (neat) $3369,1701,1597,1524,1375,1234,1193 \mathrm{~cm}^{-1}$; ${ }^{1} \mathrm{H}$ NMR $\left(500 \mathrm{MHz}, \mathrm{CDCl}_{3}\right) \delta 7.26(2 \mathrm{H}, \mathrm{s}), 5.97-6.05(1 \mathrm{H}, \mathrm{m}), 5.40\left(1 \mathrm{H}, \mathrm{dq}, J_{1,2}=17.2\right.$, $1.4 \mathrm{~Hz}), 5.22\left(1 \mathrm{H}, \mathrm{dq}, J_{1,2}=10.4,1.4 \mathrm{~Hz}\right), 4.79\left(2 \mathrm{H}, \mathrm{dt}, J_{1,2}=5.5,1.4 \mathrm{~Hz}\right), 3.97$ $(3 \mathrm{H}, \mathrm{s}) ;{ }^{13} \mathrm{C}$ NMR $\left(68 \mathrm{MHz}\right.$, acetone- $\left.d_{6}\right) \delta 166.1,151.3,133.8,126.4,117.8$, 109.9, 109.8, 65.7, 60.6 .

3,5-Di(2-nitrobenzenesulfoxy)-4-methoxybenzoic acid (22). To a solution of 21 $(400 \mathrm{mg}, 1.79 \mathrm{mmol})$ in $\mathrm{MeCN}(4 \mathrm{ml})$ were added triethylamine $(1.12 \mathrm{ml}$, $8.04 \mathrm{mmol}$ ) and 2-nitrobenzenesulfonyl chloride $(872 \mathrm{mg}, 3.93 \mathrm{mmol})$ at $0{ }^{\circ} \mathrm{C}$. The mixture was stirred at $0{ }^{\circ} \mathrm{C}$ for $1.5 \mathrm{~h}$. The reaction mixture was quenched with $2 \mathrm{M} \mathrm{HCl}$ and extracted with $\mathrm{CH}_{2} \mathrm{Cl}_{2}$. The combined organic phases were washed with brine, dried over anhydrous sodium sulfate and evaporated under reduced pressure. The residue was purified by silica gel flash column chromatography $\left(\mathrm{CH}_{2} \mathrm{Cl}_{2}\right)$ to afford crude $(954 \mathrm{mg})$ as a colorless amorphous. To a suspention of $p$-toluenesulfinic acid $(343 \mathrm{mg}, 1.92 \mathrm{mmol})$ in $\mathrm{H}_{2} \mathrm{O}(16 \mathrm{ml})$ were added a solution of the crude and tetrakis(triphenylphosphine) palladium (0) $(92.0 \mathrm{mg}, 800 \mu \mathrm{mol})$ in THF $(32 \mathrm{ml})$ at room temperature. The mixture was stirred at room temperature for $1 \mathrm{~h}$. To the resulting mixture were added $\mathrm{CH}_{2} \mathrm{Cl}_{2}$ and $\mathrm{H}_{2} \mathrm{O}$, and then organic layer was extracted with $\mathrm{H}_{2} \mathrm{O}$. The combined aqueous phases were acidified with $2 \mathrm{M} \mathrm{HCl}$ (up to pH 3.0) and extracted with ethyl acetate. The organic phase was dried over anhydrous sodium sulfate and evaporated under reduced pressure to afford 22 (743 mg, 82\%, two steps) as a colorless solid. HR-MS (FAB) calculated for $\mathrm{C}_{20} \mathrm{H}_{15} \mathrm{~N}_{2} \mathrm{O}_{13} \mathrm{~S}_{2}[\mathrm{M}+\mathrm{H}]^{+} 555.0016$, found 555.0017; IR (neat) 3026, 1703,<smiles>N#Cc1cc2c(c([N+](=O)[O-])c1)C[C@H](O)[C@H](c1cc(O)c(O)c(O)c1)O2</smiles>

53

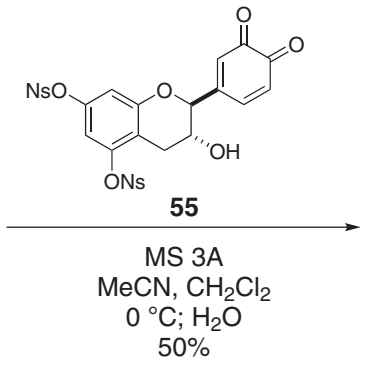

$50 \%$<smiles>[R6]Oc1cc(O)c2c(c1)O[C@H](c1cc(O)c(=O)c3c(O)c(O)cc([C@H]4O[C@H](O)Cc5c(O)cc(O[R6])cc54)c3c1)[C@H](O)C2</smiles>

$\begin{aligned} \text { 56: } \mathrm{R} & =\mathrm{Ns} \longrightarrow \begin{array}{l}\mathrm{PhSH}, \mathrm{Cs}_{2} \mathrm{CO}_{3} \\ \text { 5eCN, DMF }\end{array} \\ \mathrm{R} & =\mathrm{H} \longleftarrow\end{aligned}$

Scheme 11 Synthesis of neotheaflavin (5) by oxidative coupling reaction of 53 and 55.

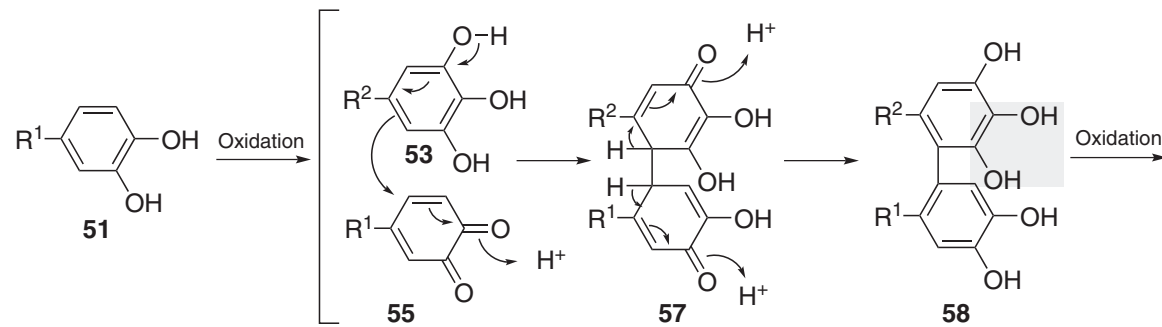<smiles>[R]c1cc(O)c(C(=O)O)c(O)c1C(=O)O</smiles>

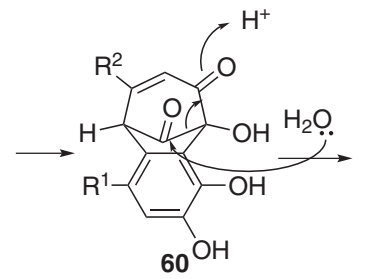<smiles>[R]C1=CC(O)=C(O)c2c(O)c(O)cc([R])c2C1([R])C(=O)O</smiles>

Oxidation

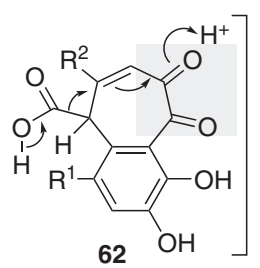<smiles>[R]c1cc(O)c(=O)c2c(O)c(O)cc([R5])c2c1</smiles>

Scheme 12 Proposed mechanism of the coupling reaction of quinone and pyrogallol according to Nakatsuka and Yanase. 
<smiles>COc1cc(O[N+](=O)[O-])cc2c1C[C@@H](O)[C@H](c1cc(O)c(O)c(O)c1)O2</smiles><smiles>CS(=O)(=O)Oc1cc2c(c(OS(=O)(=O)O)c1)C[C@@H](O)[C@@H](c1cc(O)c(=O)c3c(O)c(O)cc([C@H]4Oc5cc(O[N+](=O)[O-])cc(O[N+](=O)[O-])c5C[C@H]4O)c3c1)O2</smiles><smiles>Nc1ccccc1S</smiles><smiles>C1CCCC1</smiles><smiles>CC(C)(C)Oc1ccccc1</smiles>

$\mathrm{MeCN}$

DMF

$11 \%$

(2 steps from 53 )<smiles>C[C@H]1Cc2c(O)cc(O)cc2O[C@H]1c1cc(O)c(O)c2c(=O)c(O)cc(C3Oc4cc(O)cc(O)c4CC3O)cc12</smiles>

Scheme 13 Synthesis of theaflavin (4).

\section{Table 3 Synthesis of benzotropolone derivatives}

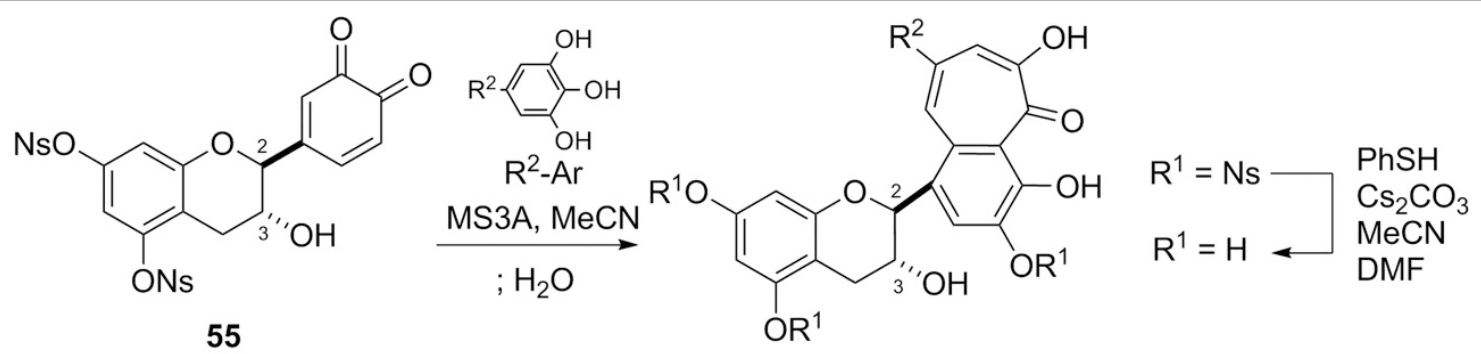

\begin{tabular}{clcr} 
Entry & \multicolumn{1}{c}{$\mathrm{R}^{2}$-Ar } & Product & $\begin{array}{r}\text { Yield } \\
\text { (2 steps fro }\end{array}$ \\
\hline 1 & $65: \mathrm{R}^{2}=\mathrm{H}$ & 67 & 13 \\
2 & $66: \mathrm{R}^{2}=\mathrm{CO}_{2} \mathrm{Me}$ & 68 & 24
\end{tabular}

1546, 1392, 1307, 1190, $1006 \mathrm{~cm}^{-1} ;{ }^{1} \mathrm{H} \quad \mathrm{NMR} \quad\left(270 \mathrm{MHz}\right.$, acetone- $\left.d_{6}\right)$ $\delta$ 7.94-8.18 (8H, m), $7.78(2 \mathrm{H}, \mathrm{s}), 3.72(3 \mathrm{H}, \mathrm{s}) ;{ }^{13} \mathrm{C}$ NMR $(68 \mathrm{MHz}$, acetone- $\left.d_{6}\right) \delta 164.8,150.5,143.4,137.7,132.6,128.5,127.0,126.2,125.6,62.7$.

Allyl 4,5-dihydroxy-3-methoxybenzoate (24). To a suspension of 20 (300 mg, $1.43 \mathrm{mmol})$ in $\mathrm{H}_{2} \mathrm{O}(20 \mathrm{ml})$ was added sodium tetraborate decahydrate $(1.40 \mathrm{~g}$, $3.67 \mathrm{mmol}$ ), then the mixture was stirred at room temperature for $1 \mathrm{~h}$. After stirring, dimethyl sulfate $(0.523 \mathrm{ml}, 5.53 \mathrm{mmol})$ and $6.5 \mathrm{M} \mathrm{NaOH}(25 \mathrm{ml})$ were added to the reaction mixture, which was stirred at room temperature for $12 \mathrm{~h}$. The reaction mixture was acidified with concentrated $\mathrm{H}_{2} \mathrm{SO}_{4}$ (up to $\mathrm{pH} 2.0$ ) and stirred at room temperature for $1 \mathrm{~h}$. The resulting mixture was poured into water and extracted with $\mathrm{CH}_{2} \mathrm{Cl}_{2}$. The organic phase was washed with brine, dried over anhydrous sodium sulfate and evaporated to afford $24(320 \mathrm{mg}$, $85 \%$ ) as a colorless solid. HR-MS (FAB) calculated for $\mathrm{C}_{11} \mathrm{H}_{12} \mathrm{O}_{5}[\mathrm{M}+\mathrm{H}]$ ${ }^{+} 224.0685$, found 224.0705; IR (neat) 3371, 1710, 1602, 1531, 1382, 1246, $1192 \mathrm{~cm}^{-1}$; ${ }^{1} \mathrm{H}$ NMR $\left(270 \mathrm{MHz}\right.$, acetone- $\left.d_{6}\right) \delta 7.23(1 \mathrm{H}, \mathrm{d}, J=2.0 \mathrm{~Hz}), 7.16$
$(1 \mathrm{H}, \mathrm{d}, J=2.0 \mathrm{~Hz}), 5.98-6.10(1 \mathrm{H}, \mathrm{m}), 5.36\left(1 \mathrm{H} \mathrm{dq}, J_{1,2}=17.1,1.5 \mathrm{~Hz}\right), 5.21$ $\left(1 \mathrm{H}, \mathrm{dq}, J_{1,2}=10.4,1.5 \mathrm{~Hz}\right), 4.73\left(2 \mathrm{H}, \mathrm{dt}, J_{1,2}=5.5,1.5 \mathrm{~Hz}\right), 3.87(3 \mathrm{H}, \mathrm{s}) ;{ }^{13} \mathrm{C}$ NMR (68 MHz, acetone- $\left.d_{6}\right) \delta 166.3,148.5,145.8,139.7,133.8,121.6,1177.8$, 111.6, 105.7, 65.6, 56.5 .

4,5-Di(2-nitrobenzenesulfoxy)-3-methoxybenzoic acid (25). In a similar manner to that used to prepare 22, treatment of $\mathbf{2 4}$ gave $\mathbf{2 5}$ (95\%, two steps) as a colorless solid. HR-MS (FAB) calculated for $\mathrm{C}_{20} \mathrm{H}_{15} \mathrm{~N}_{2} \mathrm{O}_{13} \mathrm{~S}_{2}[\mathrm{M}+\mathrm{H}]^{+} 555.0016$, found 555.0017; IR (neat) 3008, 1705, 1556, 1385, 1194, 1078, $970 \mathrm{~cm}^{-1}$; ${ }^{1} \mathrm{H}$ NMR $\left(270 \mathrm{MHz}\right.$, acetone- $\left.d_{6}\right) \delta 7.90-8.13(8 \mathrm{H}, \mathrm{m}), 7.68(1 \mathrm{H}, \mathrm{d}, J=2.0 \mathrm{~Hz})$, $7.52(1 \mathrm{H}, \mathrm{d}, J=2.0 \mathrm{~Hz}), 3.72(3 \mathrm{H}, \mathrm{s}) ;{ }^{13} \mathrm{C}$ NMR $\left(68 \mathrm{MHz}\right.$, acetone- $\left.d_{6}\right) \delta 165.4$, $154.5,143.3,137.8,137.0,133.9,133.6,132.2,131.5,130.5,128.5,126.2,125.9$, 117.2, 113.7, 57.1 .

3,4,5-Tri(2-nitrobenzenesulfoxy)benzoic acid (26). In a similar manner to that used to prepare $\mathbf{2 2}$, treatment of $\mathbf{2 0}$ gave $\mathbf{2 6}(\mathbf{8 6 \%})$ as a colorless solid. HR-MS 
(FAB) calculated for $\mathrm{C}_{25} \mathrm{H}_{15} \mathrm{~N}_{3} \mathrm{O}_{17} \mathrm{~S}_{3} \mathrm{Na}[\mathrm{M}+\mathrm{Na}]^{+} 747.9461$, found 747.9468 ; IR (neat) 3437, 1701, 1541, 1400,1305, 1193,1091, $1014 \mathrm{~cm}^{-1} ;{ }^{1} \mathrm{H}$ NMR $\left(500 \mathrm{MHz}\right.$, acetone- $\left.d_{6}\right) \delta 8.15-7.85 \quad(14 \mathrm{H}, \mathrm{m}) ;{ }^{13} \mathrm{C} \quad \mathrm{NMR} \quad(125 \mathrm{MHz}$, acetone- $\left.d_{6}\right) \delta 163.5,148.5,148.1,143.0,138.2,137.4,137.1,133.3,132.0$, $131.8,131.2,128.3,126.9,125.6,124.0$

5-((2R,3R)-3-Hydroxy-5,7-bis(2-nitrophenylsulfonyloxy)chroman-2-yl)benzene1,2,3-triyl tris(2-nitrobenzenesulfonate) (28). To a solution of 27 (100 mg, $0.327 \mathrm{mmol})$ in $\mathrm{MeCN}(20 \mathrm{ml})$ were added triethylamine $(0.453 \mathrm{ml}$, $3.27 \mathrm{mmol}$ ) and 2-nitrobenzenesulfonyl chloride $(362 \mathrm{mg}, 1.63 \mathrm{mmol})$ at $-20^{\circ} \mathrm{C}$. The mixture was stirred at $-20^{\circ} \mathrm{C}$ for $1.5 \mathrm{~h}$. The reaction mixture was quenched with $2 \mathrm{M} \mathrm{HCl}$ and extracted with ethyl acetate. The organic phase was washed with brine, dried over anhydrous sodium sulfate and evaporated under reduced pressure. The residue was purified by flash column chromatography $\left(\mathrm{CH}_{2} \mathrm{Cl}_{2}\right)$ to afford $\mathbf{2 8}(377 \mathrm{mg})$ as a colorless amorphous. HR-MS (FAB) calculated for $\mathrm{C}_{45} \mathrm{H}_{30} \mathrm{~N}_{5} \mathrm{O}_{27} \mathrm{~S}_{5}[\mathrm{M}+\mathrm{H}]^{+} 1231.9732$, found 1231.9751; $[\alpha]$ $\mathrm{D}_{20}=-5.33$ (c 1.00, acetone); IR (neat) $3406,1701,1618,1589,1542,1421$, $1388,1362,1305,1222,1193,1114,1008 \mathrm{~cm}^{-1}$; ${ }^{1} \mathrm{H}$ NMR $\left(270 \mathrm{MHz}, \mathrm{CD}_{3} \mathrm{CN}\right)$ $\delta 7.70-8.05(20 \mathrm{H}, \mathrm{m}), 7.24(2 \mathrm{H}, \mathrm{s}), 6.66(1 \mathrm{H}, \mathrm{d}, J=2.6 \mathrm{~Hz}), 6.44(1 \mathrm{H}, \mathrm{d}$, $J=2.6 \mathrm{~Hz}), 5.01(1 \mathrm{H}, \mathrm{m}), 4.05(1 \mathrm{H}, \mathrm{m}), 3.04(1 \mathrm{H}, \mathrm{br} \mathrm{s}), 2.88-2.72(2 \mathrm{H}, \mathrm{m})$; ${ }^{13} \mathrm{C}$ NMR $\left(68 \mathrm{MHz}, \mathrm{CD}_{3} \mathrm{CN}\right) \delta 156.5,149.5,149.2,148.9,148.3,143.5,141.1$, $138.0,137.9,137.7,135.2,134.1,134.1,134.0,133.8,133.1,133.0,132.8,132.6$, $129.3,128.2,127.9,127.5,126.4,126.3,122.1,118.3,115.7,110.7,109.9,78.3$, $64.2,29.3$.

5-(2R,3R)-5,7-Bis(2-nitrophenylsulfonyloxy)-2-(3,4,5-tris(2-nitrophenylsulfonyloxy) phenyl) chroman-3-yl-3-methoxy-4,5-bis(2-nitrophenylsulfonyloxy)benzoate (29).

28 (700 mg, $0.568 \mathrm{mmol}$ ), 25 (628 mg, $1.14 \mathrm{mmol}$ ), EDCI $(327 \mathrm{mg}$, $1.70 \mathrm{mmol})$ and DMAP $(13.9 \mathrm{mg}, 0.114 \mathrm{mmol})$ were dissolved in $\mathrm{CH}_{3} \mathrm{CN}$ $(3 \mathrm{ml})$ and stirred at room temperature for $16 \mathrm{~h}$. The resulting mixture was quenched with saturated $\mathrm{NH}_{4} \mathrm{Cl}$ and extracted with $\mathrm{CH}_{2} \mathrm{Cl}_{2}$. The organic phase was dried over anhydrous sodium sulfate and evaporated under reduced pressure. The residue was purified by silica gel flash column chromatography (25\% hexane in $\mathrm{CH}_{2} \mathrm{Cl}_{2}$ ) to afford 29 (925 mg, 92\%) as a colorless amorphous.

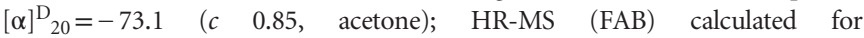
$\mathrm{C}_{65} \mathrm{H}_{41} \mathrm{~N}_{7} \mathrm{O}_{39} \mathrm{~S}_{7} \mathrm{Na}[\mathrm{M}+\mathrm{Na}]^{+} 1789.9383$, found 1789.9386; IR (neat) 1708, $1618,1593,1545,1419,1394,1366,1305,1193,1121,1001 \mathrm{~cm}^{-1} ;{ }^{1} \mathrm{H}$ NMR $\left(270 \mathrm{MHz}\right.$, acetone- $\left.d_{6}\right) \delta 7.80-8.14(28 \mathrm{H}, \mathrm{m}), 7.54(2 \mathrm{H}, \mathrm{s}), 7.34(1 \mathrm{H}, \mathrm{d}$, $J=2.0 \mathrm{~Hz}), 7.23(1 \mathrm{H}, \mathrm{d}, J=2.0 \mathrm{~Hz}), 6.97(1 \mathrm{H}, \mathrm{d}, J=2.6 \mathrm{~Hz}), 6.66(1 \mathrm{H}, \mathrm{d}$, $J=2.6 \mathrm{~Hz}), 5.73-5.78(1 \mathrm{H}, \mathrm{m}), 5.65(1 \mathrm{H}, \mathrm{s}), 3.59(3 \mathrm{H}, \mathrm{s}), 3.30(1 \mathrm{H}, \mathrm{dd}$, $\left.J_{1,2}=17.5,4.3 \mathrm{~Hz}\right), 3.23\left(1 \mathrm{H}, \mathrm{dd}, \mathrm{J}_{1,2}=17.5,3.3 \mathrm{~Hz}\right) ;{ }^{13} \mathrm{C}$ NMR $(68 \mathrm{MHz}$, acetone- $\left.\mathrm{d}_{6}\right) \delta 162.1,155.6,150.2,148.7,148.4,147.8,142.6,141.0,140.8,138.5$, $137.1,137.0,136.9,133.1,133.0,133.0,132.9,132.3,132.0,131.9,131.8,127.6$, $127.5,127.4,127.2,126.9,126.7,125.4,125.4,125.3,124.8,124.7,124.7,122.5$, $113.7,110.4,109.7,76.4,67.7,62.0,26.0$.

(2R,3R)-5,7-Dihydroxy-2-(3,4,5-trihydroxyphenyl)chroman-3-yl 4,5-dihydroxy-3methoxybenzoate (2). To a suspension of $\mathrm{Cs}_{2} \mathrm{CO}_{3}(3.61 \mathrm{~g}, 11.3 \mathrm{mmol})$ in $\mathrm{MeCN}(6 \mathrm{ml})$ were added thiophenol $(1.16 \mathrm{ml}, 5.75 \mathrm{mmol})$ and $29(800 \mathrm{mg}$, $0.452 \mathrm{mmol}$ ) at $0{ }^{\circ} \mathrm{C}$. The mixture was stirred at room temperature for $3.5 \mathrm{~h}$. The reaction was quenched with sat. $\mathrm{NH}_{4} \mathrm{Cl}$ and extracted with ethyl acetate. The organic phase was dried over anhydrous sodium sulfate and evaporated under reduced pressure. The residue was purified by silica gel flash column chromatography $\left(7 \% \mathrm{MeOH}\right.$ in $\left.\mathrm{CH}_{2} \mathrm{Cl}_{2}\right)$ to afford $2(174 \mathrm{mg}, 81 \%)$ as a colorless amorphous. HR-MS (FAB) calculated for $\mathrm{C}_{23} \mathrm{H}_{20} \mathrm{O}_{11} \mathrm{Na}[\mathrm{M}+\mathrm{Na}]$ ${ }^{+} 495.0904$, found $495.0873 ;[\alpha]^{\mathrm{D}}{ }_{20}=-186.2\left(c 1.00,50 \%\right.$ acetone in $\left.\mathrm{H}_{2} \mathrm{O}\right)$; IR (neat) $3327,1701,1610,1340,1229 \mathrm{~cm}^{-1}$; ${ }^{1} \mathrm{H}$ NMR $\left(270 \mathrm{MHz}, \mathrm{CD}_{3} \mathrm{OD}\right) \delta$ $7.05(1 \mathrm{H}, \mathrm{d}, J=1.9 \mathrm{~Hz}), 7.01(1 \mathrm{H}, \mathrm{d}, J=1.9 \mathrm{~Hz}), 6.50(2 \mathrm{H}, \mathrm{s}), 5.96(1 \mathrm{H}, \mathrm{d}$, $J=2.6 \mathrm{~Hz}), 5.95(1 \mathrm{H}, \mathrm{d}, J=2.6 \mathrm{~Hz}), 5.49(1 \mathrm{H}, \mathrm{m}), 5.00(1 \mathrm{H}, \mathrm{s}), 3.81(3 \mathrm{H}, \mathrm{s})$, $2.99\left(1 \mathrm{H}, \mathrm{dd}, J_{1,2}=17.5,4.3 \mathrm{~Hz}\right), 2.86\left(1 \mathrm{H}, \mathrm{dd}, J_{1,2}=17.5,3.0 \mathrm{~Hz}\right) ;{ }^{13} \mathrm{C} \mathrm{NMR}$ $\left(68 \mathrm{MHz}, \mathrm{CD}_{3} \mathrm{OD}\right) \delta 167.7,157.9,157.8,157.2,149.0,146.8,146.0,140.6$, $133.7,130.9,121.5,111.9,106.8,106.3,99.4,96.5,95.8,78.5,70.4,56.6,26.6$

5-(2R,3R)-5,7-Bis(2-nitrophenylsulfonyloxy)-2-(3,4,5-tris(2-nitrophenylsulfonyloxy) phenyl) chroman-3-yl-4-methoxy-3,5-bis(2-nitrophenylsulfonyloxy)benzoate (30).

In a similar manner to that used to prepare $\mathbf{2 9}$, treatment of $\mathbf{2 8}$ with $\mathbf{2 2}$ gave $30(97 \%)$ as a colorless amorphous. HR-MS (FAB) calculated for $\mathrm{C}_{65} \mathrm{H}_{41} \mathrm{~N}_{7} \mathrm{O}_{39} \mathrm{~S}_{7} \mathrm{Na} \quad[\mathrm{M}+\mathrm{Na}]^{+} 1789.9383$, found 1789.9386; $[\alpha]^{\mathrm{D}}{ }_{20}=-73.1$ (c 0.85, acetone); IR (neat) 1708, 1618, 1593, 1545, 1419, 1394, 1366, 1305, $1193,1121,1001 \mathrm{~cm}^{-1} ;{ }^{1} \mathrm{H}$ NMR $\left(500 \mathrm{MHz}\right.$, acetone- $\left.d_{6}\right) \delta 7.80-8.14(28 \mathrm{H}$, m), $7.57(2 \mathrm{H}, \mathrm{s}), 7.46(2 \mathrm{H}, \mathrm{s}), 6.98(1 \mathrm{H}, \mathrm{d}, J=2.6 \mathrm{~Hz}), 6.66(1 \mathrm{H}, \mathrm{d}, J=2.6 \mathrm{~Hz})$, $5.74(1 \mathrm{H}, \mathrm{m}), 5.60(1 \mathrm{H}, \mathrm{s}), 3.63(3 \mathrm{H}, \mathrm{s}), 3.28\left(1 \mathrm{H}, \mathrm{dd}, J_{1,2}=17.5,4.3 \mathrm{~Hz}\right), 3.10$ $\left(1 \mathrm{H}, \mathrm{dd}, J_{1,2}=17.5,3.3 \mathrm{~Hz}\right) ;{ }^{13} \mathrm{C}$ NMR $\left(126 \mathrm{MHz}\right.$, acetone- $\left.d_{6}\right) \delta 162.2,155.3$, $150.2,148.8,148.4,148.2,147.7,142.9,142.7,138.7,137.2,136.9,136.9,134.4$, $133.2,133.1,133.1,133.0,132.3,132.1,132.0,131.7,131.6,128.3,127.8,127.1$, $126.9,126.6,125.5,125.5,125.4,124.9,124.8,121.2,113.8,110.4,109.9,75.9$, $67.4,61.9,26.0$.

(2R,3R)-5,7-Dihydroxy-2-(3,4,5-trihydroxyphenyl)chroman-3-yl 3,5-dihydroxy-4methoxybenzoate (3). In a similar manner to that used to prepare 2 , treatment of $\mathbf{3 0}$ gave $3(81 \%)$ as a colorless amorphous. HR-MS (FAB) calculated for $\mathrm{C}_{23} \mathrm{H}_{20} \mathrm{O}_{11} \mathrm{Na}[\mathrm{M}+\mathrm{Na}]^{+} 495.0904$, found 495.0896; $[\alpha]^{\mathrm{D}}{ }_{20}=-171.5$ (c 1.20, $50 \%$ acetone in $\mathrm{H}_{2} \mathrm{O}$ ); IR (neat) $3367,1701,1604,1348,1236 \mathrm{~cm}^{-1}$; ${ }^{1} \mathrm{H}$ NMR $\left(270 \mathrm{MHz}, \mathrm{CD}_{3} \mathrm{OD}\right) \delta 6.91(2 \mathrm{H}, \mathrm{s}), 6.49(2 \mathrm{H}, \mathrm{s}), 5.95(2 \mathrm{H}, \mathrm{s}), 5.53(1 \mathrm{H}, \mathrm{m})$, $4.97(1 \mathrm{H}, \mathrm{s}), 3.81(3 \mathrm{H}, \mathrm{s}), 2.99\left(1 \mathrm{H}, \mathrm{dd}, J_{1,2}=17.5,4.6 \mathrm{~Hz}\right), 2.84(1 \mathrm{H}, \mathrm{dd}$, $\left.J_{1,2}=17.5,2.3 \mathrm{~Hz}\right) ;{ }^{13} \mathrm{C} \mathrm{NMR}\left(125 \mathrm{MHz}, \mathrm{CD}_{3} \mathrm{OD}\right) \delta 167.2,157.9,157.8,157.2$, $151.5,146.7,141.1,133.8,130.7,126.6,110.3,106.8,99.3,96.5,95.9,78.5,70.3$, $60.7,26.8$

(2R,3R)-2-(3,4-Dihydroxy-5-(2-nitrophenylsulfonyloxy)phenyl)-3-hydroxychroman5,7-diyl bis(2-nitrobenzenesulfonate) (32). To a solution of boric acid (4.64 g, $80.3 \mathrm{mmol})$ and $\mathrm{NaOH}(800 \mathrm{mg})$ in $\mathrm{H}_{2} \mathrm{O}(160 \mathrm{ml})$ was added $27(492 \mathrm{mg}$, $1.61 \mathrm{mmol}$ ) and the resulting solution was adjusted to $\mathrm{pH}$ 9.0. Then, 2-nitrobenzenesulfonyl chloride $(1.06 \mathrm{~g}, 4.83 \mathrm{mmol})$ in toluene $(16 \mathrm{ml})$ was added dropwise over $30 \mathrm{~min}$ and stirred at room temperature for $3 \mathrm{~h}$. The reaction was quenched with $2 \mathrm{M} \mathrm{HCl}$ and extracted with ethyl acetate. The organic phase was dried over sodium sulfate and evaporated under reduced pressure. The residue was purified by silica gel flash column chromatography (1-3\% $\mathrm{MeOH}$ in $\mathrm{CH}_{2} \mathrm{Cl}_{2}$ ) to afford $32(560 \mathrm{mg}, 40 \%)$ as a pale yellow solid. HR-MS (FAB) calculated for $\mathrm{C}_{33} \mathrm{H}_{23} \mathrm{~N}_{3} \mathrm{O}_{19} \mathrm{~S}_{3} \mathrm{Na}[\mathrm{M}+\mathrm{Na}]^{+} 883.9986$, found 883.9965; $[\alpha]^{\mathrm{D}}{ }_{20}=+10.6$ (c 1.28, acetone); IR (neat) 3422, 1701, 1618, 1591, $1541,1440,1388,1358,1305,1191,1111,995 \mathrm{~cm}^{-1} ;{ }^{1} \mathrm{H}$ NMR $(270 \mathrm{MHz}$, acetone- $\left.d_{6}\right) \delta 7.80-8.20(12 \mathrm{H}, \mathrm{m}), 6.99(1 \mathrm{H}, \mathrm{d}, J=2.3 \mathrm{~Hz}), 6.71(1 \mathrm{H}, \mathrm{d}$, $J=2.3 \mathrm{~Hz}), 6.62(1 \mathrm{H}, \mathrm{d}, J=2.3 \mathrm{~Hz}), 6.50(1 \mathrm{H}, \mathrm{d}, J=2.3 \mathrm{~Hz}), 4.99(1 \mathrm{H}, \mathrm{m})$, $4.22(1 \mathrm{H}, \mathrm{m}), 2.80-2.98(2 \mathrm{H}, \mathrm{m}) ;{ }^{13} \mathrm{C}$ NMR $\left(68 \mathrm{MHz}\right.$, acetone- $\left.d_{6}\right) \delta 157.3$, $149.5,148.2,147.3,138.9,138.2,137.8,137.0,133.9,133.6,133.3,133.0,132.9$, $132.6,130.2,129.5,128.4,127.7,126.3,126.2,125.8,116.0,114.1,113.4,110.3$, $109.1,79.4,64.8,29.6$.

(2R,3R)-2-(3-tert-Butyldiphenylsilyloxy-4-hydroxy-5-(2-nitrophenylsulfonyloxy) phenyl)-3-hydroxychroman-5,7-diyl bis(2-nitrobenzenesulfonate) (33). Tо a solution of $32(200 \mathrm{mg}, 0.182 \mathrm{mmol})$ in $\mathrm{MeCN}(0.4 \mathrm{ml})$ were added triethylamine $(0.196 \mathrm{ml}, 1.09 \mathrm{mmol})$ and TBDPSCl $(197 \mathrm{mg}, 0.545 \mathrm{mmol})$ at $-20^{\circ} \mathrm{C}$, then the resulting solution was stirred at $-20^{\circ} \mathrm{C}$ for $3 \mathrm{~h}$. The reaction mixture was quenched with $2 \mathrm{M} \mathrm{HCl}$ and extracted with $\mathrm{CH}_{2} \mathrm{Cl}_{2}$. The combined organic phases were dried over sodium sulfate and evaporated under reduced pressure. The residue was purified by silica gel flash column chromatography (50\% ethyl acetate in hexane) to afford $33(211 \mathrm{mg}, 83 \%)$ as a pale yellow solid. HR-MS (FAB) calculated for $\mathrm{C}_{33} \mathrm{H}_{23} \mathrm{~N}_{3} \mathrm{O}_{19} \mathrm{~S}_{3} \mathrm{Na}[\mathrm{M}+\mathrm{Na}]^{+} 1122.1163$, found 1122.1191; $[\alpha]^{\mathrm{D}}{ }_{20}=-0.68$ (c 1.35, acetone); IR (neat) $3448,1707,1618,1591,1544,1441$, $1388,1364,1305,1193,1112,997 \mathrm{~cm}^{-1} ;{ }^{1} \mathrm{H}$ NMR $\left(270 \mathrm{MHz}, \mathrm{CDCl}_{3}\right)$ $\delta, 7.36-8.13(23 \mathrm{H}, \mathrm{m}), 6.77(1 \mathrm{H}, \mathrm{d}, J=2.0 \mathrm{~Hz}), 6.51(1 \mathrm{H}, \mathrm{d}, J=2.0 \mathrm{~Hz})$, $6.43(1 \mathrm{H}, \mathrm{d}, J=2.0 \mathrm{~Hz}), 6.37(1 \mathrm{H}, \mathrm{d}, J=2.0 \mathrm{~Hz}), 4.58(1 \mathrm{H}, \mathrm{m}), 3.73(1 \mathrm{H}, \mathrm{m})$, 2.82-2.86 (2H, m), $1.09(9 \mathrm{H}, \mathrm{s}) ;{ }^{13} \mathrm{C}$ NMR $\left(68 \mathrm{MHz}, \mathrm{CDCl}_{3}\right) \delta 155.4,148.3$, $147.1,144.4,140.1,136.4,135.9,135.8,135.5,135.4,135.3,132.6,132.3,132.3$, $132.1,130.6,130.6,128.1,127.7,125.0,124.9,124.8,116.0,114.3,113.9,110.0$, $109.1,64.6,28.0,26.6,19.5$.

(2R,3R)-2-(3-tert-Butyldiphenylsilyloxy-4-methoxy-5-(2-nitrophenylsulfonyloxy) phenyl)-3-hydroxychroman-5,7-diyl bis(2-nitrobenzenesulfonate) (35). To a solution of $33(125 \mathrm{mg}, 0.110 \mathrm{mmol})$ in $\mathrm{MeCN}(1 \mathrm{ml})$ were added diazomethane $\left(\mathrm{Et}_{2} \mathrm{O}\right.$ solution) until color of the solution became yellow at $0{ }^{\circ} \mathrm{C}$. The resulting solution was stirred at $0^{\circ} \mathrm{C}$ for $10 \mathrm{~min}$. The reaction was quenched with acetic acid and evaporated under reduced pressure. The residue was purified by silica gel flash column chromatography $(50 \%$ ethyl acetate in hexane) to afford $35(115 \mathrm{mg}, 91 \%)$ as a pale yellow solid. HR-MS (FAB) 
calculated for $\mathrm{C}_{33} \mathrm{H}_{23} \mathrm{~N}_{3} \mathrm{O}_{19} \mathrm{~S}_{3} \mathrm{Na} \quad[\mathrm{M}+\mathrm{Na}]^{+} 1136.1320$, found 1136.1298; $[\alpha]^{\mathrm{D}_{20}}=-9.17$ (c 1.25, acetone); IR (neat) 3420, 1709, 1618, 1591, 1550, 1441, 1391, 1360, 1308, 1196, 1113, $997 \mathrm{~cm}^{-1}$; ${ }^{1} \mathrm{H}$ NMR $\left(500 \mathrm{MHz}, \mathrm{CDCl}_{3}\right) \delta$, 8.08-7.36 $(22 \mathrm{H}, \mathrm{m}), 6.71(1 \mathrm{H}, \mathrm{d}, J=2.4 \mathrm{~Hz}), 6.50(1 \mathrm{H}, \mathrm{d}, J=2.4 \mathrm{~Hz}), 6.43$ $(1 \mathrm{H}, \mathrm{d}, J=2.4 \mathrm{~Hz}), 6.40(1 \mathrm{H}, \mathrm{d}, J=2.4 \mathrm{~Hz}), 4.58(1 \mathrm{H}, \mathrm{m}), 3.84(3 \mathrm{H}, \mathrm{s}), 3.71$ $(1 \mathrm{H}, \mathrm{m}), 2.89\left(1 \mathrm{H}, \mathrm{dd}, \mathrm{J}_{1,2}=17.7,2.4 \mathrm{~Hz}\right), 2.89\left(1 \mathrm{H}, \mathrm{dd}, \mathrm{J}_{1,2}=17.7,4.0 \mathrm{~Hz}\right)$, $1.09(9 \mathrm{H}, \mathrm{s}) ;{ }^{13} \mathrm{C}$ NMR $\left(68 \mathrm{MHz}, \mathrm{CDCl}_{3}\right) \delta 155.3,150.3,148.6,148.3,147.1$, $143.1,135.9,135.8,135.5,135.4,135.3,132.6,132.3,132.3,132.1,132.0,131.9$, $131.8,130.4,130.3,128.2,128.0,128.0,125.0,124.9,124.7,117.8,114.1,113.9$, $110.0,109.1,64.5,61.3,28.0,26.4,19.5$.

(2R,3R)-2-(3-tert-Butyldiphenylsilyloxy-4-methoxy-5-(2-nitrophenylsulfonyloxy) phenyl)-5,7-bis(2-nitrophenylsulfonyloxy)-chroman-3-yl-3,4,5-tris(2-nitrophenylsulfonyloxy)benzoate (69). In a similar manner to that used to prepare 29, treatment of 35 with 26 gave $69(86 \%)$ as a colorless solid. HR-MS (FAB) calculated for $\mathrm{C}_{75} \mathrm{H}_{56} \mathrm{~N}_{6} \mathrm{O}_{35} \mathrm{SiS}_{6} \mathrm{Na}[\mathrm{M}+\mathrm{Na}]^{+} 1843.0778$, found 1843.0771; $[\alpha]_{20}^{\mathrm{D}_{20}}=-42.3$ (c 1.00, acetone); IR (neat) 1711, 1618, 1591, 1545, 1442, 1391, 1364, 1308, 1193, 1114, $1005 \mathrm{~cm}^{-1} ;{ }^{1} \mathrm{H}$ NMR $\left(500 \mathrm{MHz}, \mathrm{CDCl}_{3}\right)$ $\delta 7.34-8.03(34 \mathrm{H}, \mathrm{m}), 6.68(1 \mathrm{H}, \mathrm{d}, J=1.9 \mathrm{~Hz}), 6.67(1 \mathrm{H}, \mathrm{d}, J=1.9 \mathrm{~Hz}), 6.60$ $(1 \mathrm{H}, \mathrm{d}, J=2.0 \mathrm{~Hz}), 6.26(1 \mathrm{H}, \mathrm{d}, J=2.0 \mathrm{~Hz}), 5.04(1 \mathrm{H}, \mathrm{m}), 4.64(1 \mathrm{H}, \mathrm{m}), 3.70$ $(3 \mathrm{H}, \mathrm{s}), 2.95\left(1 \mathrm{H}, \mathrm{dd}, J_{1,2}=18.0,4.6 \mathrm{~Hz}\right), 2.80\left(1 \mathrm{H}, \mathrm{dd}, J_{1,2}=18.0,1.5 \mathrm{~Hz}\right), 1.02$ $(9 \mathrm{H}, \mathrm{s}) ;{ }^{13} \mathrm{C}$ NMR $\left(68 \mathrm{MHz}, \mathrm{CDCl}_{3}\right) \delta 161.4,155.1,150.1,148.6,148.4,148.2$, $148.2,148.0,147.4,143.6,143.1,143.1,139.0,136.3,136.3,136.1,135.9,135.4$, $135.3,133.0,132.9,132.7,132.6,132.3,132.1,131.9,131.9,131.7,131.1,130.4$, $130.4,129.2,128.9,128.0,128.0,127.8,127.7,127.4,125.4,125.1,125.0,124.8$, $124.1,117.6,114.3,112.4,110.2,110.0,67.4,61.3,26.5,26.3,19.5$.

(2R,3R)-2-(3-Hydroxy-4-methoxy-5-(2-nitrophenylsulfonyloxy)phenyl)-5,7-bis(2nitrophenylsulfonyloxy)-chroman-3-yl-3,4,5-tris(2-nitrophenylsulfonyloxy)benzoate (70). To a solution of $69(400 \mathrm{mg}, 0.220 \mathrm{mmol})$ and acetic acid $(0.0377 \mathrm{ml}$, $0.661 \mathrm{mmol})$ in tetrahydrofuran (THF) $(4.6 \mathrm{ml})$ was added TBAF $(1.0 \mathrm{M}$ solution in THF, $0.266 \mathrm{ml}, 0.266 \mathrm{mmol})$ at $0{ }^{\circ} \mathrm{C}$, then the resulting solution was stirred at $0{ }^{\circ} \mathrm{C}$ for $15 \mathrm{~min}$. The reaction mixture was poured into water and extracted with $\mathrm{CH}_{2} \mathrm{Cl}_{2}$. The organic phase was dried over anhydrous sodium sulfate and evaporated under reduced pressure to afford crude $70(320 \mathrm{mg})$ as a colorless solid. The crude 70 was used in the next step without further purification. ${ }^{1} \mathrm{H}$ NMR $\left(500 \mathrm{MHz}\right.$, acetone- $\left.d_{6}\right) \delta 7.62-8.07(24 \mathrm{H}, \mathrm{m}), 7.57$ $(2 \mathrm{H}, \mathrm{s}), 6.83(1 \mathrm{H}, \mathrm{d}, J=1.8 \mathrm{~Hz}), 6.82(1 \mathrm{H}, \mathrm{d}, J=1.8 \mathrm{~Hz}), 6.69(1 \mathrm{H}, \mathrm{d}$, $J=1.8 \mathrm{~Hz}), 6.65(1 \mathrm{H}, \mathrm{d}, J=1.8 \mathrm{~Hz}), 5.50(1 \mathrm{H}, \mathrm{m}), 4.06(1 \mathrm{H}, \mathrm{m}), 3.80(3 \mathrm{H}, \mathrm{s})$, $3.19\left(1 \mathrm{H}, \mathrm{dd}, J_{1,2}=18.3,4.3 \mathrm{~Hz}\right), 3.03\left(1 \mathrm{H}, \mathrm{dd}, J_{1,2}=18.3,4.3 \mathrm{~Hz}\right)$

(2R,3R)-2-(3,5-Dihydroxy-4-methoxyphenyl)-5,7-dihydroxychroman-3-yl 3,4,5trihydroxybenzoate (7). In a similar manner to that used to prepare 2, treatment of crude 70 gave 7 (77\%, 2 steps) as a colorless amorphous. HR-MS (FAB) calculated for $\mathrm{C}_{23} \mathrm{H}_{20} \mathrm{O}_{11} \mathrm{Na}[\mathrm{M}+\mathrm{Na}]^{+}$495.0904, found 495.0942; $[\alpha]^{\mathrm{D}}{ }_{20}=-147.6\left(c 1.00,50 \%\right.$ acetone in $\left.\mathrm{H}_{2} \mathrm{O}\right)$; IR (neat) 3346, $1701,1608,1357,1234 \mathrm{~cm}^{-1} ;{ }^{1} \mathrm{H}$ NMR $\left(270 \mathrm{MHz}, \mathrm{CD}_{3} \mathrm{OD}\right) \delta 6.84(2 \mathrm{H}, \mathrm{s})$, $6.43(2 \mathrm{H}, \mathrm{s}), 5.87(2 \mathrm{H}, \mathrm{s}), 5.44(1 \mathrm{H}, \mathrm{m}), 4.89(1 \mathrm{H}, \mathrm{m}), 3.66(3 \mathrm{H}, \mathrm{s}), 2.90(1 \mathrm{H}$, dd, $\left.J_{1,2}=17.1,4.6 \mathrm{~Hz}\right), 2.75\left(1 \mathrm{H}, \mathrm{dd}, J_{1,2}=17.1,2.3 \mathrm{~Hz}\right) ;{ }^{13} \mathrm{C} \mathrm{NMR}(68 \mathrm{MHz}$, $\left.\mathrm{CD}_{3} \mathrm{OD}\right) \delta 167.6,157.8,157.0,151.3,146.2,136.3,136.0,135.8,129.8,121.5$, $110.3,107.1,99.4,96.6,95.9,78.4,69.8,60.8,26.8$.

(2R,3R)-2-(3-tert-Butyldiphenylsilyloxy-4,5-bis(2-nitrophenylsulfonyloxy)phenyl)3-hydroxychroman-5,7-diyl bis(2-nitrobenzenesulfonate) (71). In a similar manner to that used to prepare 28, treatment of 33 gave $71(95 \%)$ as a colorless amorphous. HR-MS (FAB) calculated for $\mathrm{C}_{56} \mathrm{H}_{44} \mathrm{~N}_{4} \mathrm{O}_{23} \mathrm{SiS}_{4} \mathrm{Na}$ $[\mathrm{M}+\mathrm{Na}]^{+} 1307.0946$, found 1307.0963; $[\alpha]^{\mathrm{D}}{ }_{20}=-9.25$ (c 0.84, acetone); IR (neat) 1709, 1618,1591,1548, 1440,1391, 1360, 1306, 1196, 1113, $997 \mathrm{~cm}^{-1}$; ${ }^{1} \mathrm{H}$ NMR $\left(500 \mathrm{MHz}, \mathrm{CDCl}_{3}\right) \delta, 7.36-8.13(26 \mathrm{H}, \mathrm{m}), 6.60(1 \mathrm{H}, \mathrm{d}, J=2.0 \mathrm{~Hz})$, $6.43(1 \mathrm{H}, \mathrm{d}, J=2.0 \mathrm{~Hz}), 6.42(1 \mathrm{H}, \mathrm{d}, J=2.0 \mathrm{~Hz}), 6.35(1 \mathrm{H}, \mathrm{d}, J=2.0 \mathrm{~Hz}), 4.51$ $(1 \mathrm{H}, \mathrm{m}), 3.58(1 \mathrm{H}, \mathrm{m}), 2.88(2 \mathrm{H}, \mathrm{m}), 2.76(1 \mathrm{H}, \mathrm{dd}, J=17.5,4.0 \mathrm{~Hz}), 1.08$ $(9 \mathrm{H}, \mathrm{s}) ;{ }^{13} \mathrm{C} \mathrm{NMR}\left(125 \mathrm{MHz}, \mathrm{CDCl}_{3}\right) \delta 154.9,150.7,148.5,148.3,148.3,148.2$, $146.9,142.4,136.5,136.0,136.0,135.9,135.5,135.4,135.3,132.8,132.6,132.6$, $132.4,132.4,132.2,132.1,132.0,131.7,131.2,131.0,130.5,130.4,130.1,128.0$, 127.5, 125.1, 124.9, 124.6, 117.5, 113.8, 112.6, 109.9, 64.2, 60.4, 27.9, 26.0, 19.4.

(2R,3R)-2-(3-Hydroxy-4,5-bis(2-nitrophenylsulfonyloxy)phenyl)-3-hydroxychroman5,7-diyl bis(2-nitrobenzenesulfonate) (36). In a similar manner to that used to prepare 70, treatment of $\mathbf{7 1}$ gave $\mathbf{3 6}(86 \%)$ as a colorless amorphous. HR-MS (FAB) calculated for $\mathrm{C}_{39} \mathrm{H}_{26} \mathrm{~N}_{4} \mathrm{O}_{23} \mathrm{~S}_{4} \mathrm{Na}[\mathrm{M}+\mathrm{Na}]^{+} 1068.9768$, found 1068.9788; $[\alpha]^{\mathrm{D}}{ }_{20}=-1.41$ (c 1.80, acetone); IR (neat) $3429,1701,1612,1591,1543,1438$, $1389,1364,1194,1113,999 \mathrm{~cm}^{-1} ;{ }^{1} \mathrm{H}$ NMR $\left(270 \mathrm{MHz}\right.$, acetone- $\left.d_{6}\right) \delta$, 8.13-7.86 (16H, m), $7.17(1 \mathrm{H}, \mathrm{d}, J=2.0 \mathrm{~Hz}), 6.78(1 \mathrm{H}, \mathrm{d}, J=2.0 \mathrm{~Hz}), 6.74$ $(1 \mathrm{H}, \mathrm{d}, J=2.0 \mathrm{~Hz}), 6.50(1 \mathrm{H}, \mathrm{d}, J=2.0 \mathrm{~Hz}), 5.12(1 \mathrm{H}, \mathrm{m}), 4.29(1 \mathrm{H}, \mathrm{m})$, $2.98-2.90(2 \mathrm{H}, \mathrm{m}) ;{ }^{13} \mathrm{C}$ NMR $\left(125 \mathrm{MHz}\right.$, acetone- $\left.d_{6}\right) \delta 156.2,151.6,148.7$, $148.6,148.5,148.4,147.4,142.3,139.2,137.1,136.8,136.0,133.1,132.9,132.9$, $132.8,132.2,132.2,132.1,131.9,131.4,130.1,130.0,127.8,127.4,126.8,125.5$, 125.5, 125.3, 125.1, 115.2, 114.9, 112.2, 109.7, 108.6, 78.3, 63.7, 29.8.

(2R,3R)-2-(3-Methoxy-4,5-bis(2-nitrophenylsulfonyloxy)phenyl)-3-hydroxychroman5,7-diyl bis(2-nitrobenzenesulfonate) (37). In a similar manner to that used to prepare 35, treatment of $\mathbf{3 6}$ gave $\mathbf{3 7}(91 \%)$ as a colorless amorphous. HR-MS (FAB) calculated for $\mathrm{C}_{40} \mathrm{H}_{28} \mathrm{~N}_{4} \mathrm{O}_{23} \mathrm{~S}_{4} \mathrm{Na}[\mathrm{M}+\mathrm{Na}]^{+} 1082.9925$, found 1082.9946; $[\alpha]^{\mathrm{D}}{ }_{20}=-3.18$ (c 0.56, acetone); IR (neat) 3451, 1701, 1618, 1591, 1541, 1439, $1389, \quad 1356, \quad 1306,1194,1113,999 \mathrm{~cm}^{-1} ; \quad{ }^{1} \mathrm{H} \quad \mathrm{NMR} \quad(270 \mathrm{MHz}$, acetone- $\left.d_{6}\right) \delta 8.13-7.90(16 \mathrm{H}, \mathrm{m}), 7.30(1 \mathrm{H}, \mathrm{d}, J=2.0 \mathrm{~Hz}), 6.94(1 \mathrm{H}, \mathrm{d}$, $J=2.0 \mathrm{~Hz}), 6.77(1 \mathrm{H}, \mathrm{d}, J=2.6 \mathrm{~Hz}), 6.51(1 \mathrm{H}, \mathrm{d}, J=2.6 \mathrm{~Hz}), 5.21(1 \mathrm{H}, \mathrm{m})$, $4.37(1 \mathrm{H}, \mathrm{m}), 3.64(3 \mathrm{H}, \mathrm{s}), 2.98-2.94(2 \mathrm{H}, \mathrm{m}) ;{ }^{13} \mathrm{C}$ NMR $\left(68 \mathrm{MHz}\right.$, acetone- $\left.d_{6}\right)$ $\delta 156.2,153.3,148.7,148.6,148.5,148.3,147.4,142.2,139.6,137.1,137.0$, $136.8,136.0,133.1,133.0,132.9,132.7,132.2,132.0,131.9,131.3,130.8,130.0$, $127.9,127.5,126.8,125.5,125.5,125.4,125.1,115.1,113.4,110.7,109.8,108.7$, $78.5,63.6,54.2,29.8$.

\section{(2R,3R)-2-(3-Methoxy-4,5-bis(2-nitrophenylsulfonyloxy)phenyl)-5,7-bis(2-}

nitrophenylsulfonyloxy)-chroman-3-yl 3-methoxy-4,5-bis(2-nitrophenylsulfonyloxy) benzoate (72). 37 (20.0 mg, $0.0188 \mathrm{mmol}), 25$ (20.9 mg, $0.0377 \mathrm{mmol})$, EDCI $(10.8 \mathrm{mg}, 0.0563 \mathrm{mmol})$, DMAP $(0.2 \mathrm{mg}, 1.9 \mu \mathrm{mol})$ were dissolved in $\mathrm{MeCN}$ $(0.25 \mathrm{ml})$ and the resulting solution was stirred at room temperature for $17 \mathrm{~h}$. Then, the reaction mixture was quenched with saturated $\mathrm{NH}_{4} \mathrm{Cl}$ and extracted with $\mathrm{CH}_{2} \mathrm{Cl}_{2}$. The organic phase was dried over anhydrous sodium sulfate and evaporated under reduced pressure to afford crude 72 as a colorless solid. The crude 72 was used in the next step without further purification. ${ }^{1} \mathrm{H}$ NMR $\left(270 \mathrm{MHz}\right.$, acetone- $\left.d_{6}\right) \delta, 8.17-7.81(28 \mathrm{H}, \mathrm{m}, 28 \mathrm{H}), 7.37(1 \mathrm{H}, \mathrm{d}, J=2.0 \mathrm{~Hz}$, $1 \mathrm{H}), 7.31(1 \mathrm{H}, \mathrm{d}, J=2.0 \mathrm{~Hz}), 7.24(1 \mathrm{H}, \mathrm{d}, J=2.0 \mathrm{~Hz}), 7.04(1 \mathrm{H}, \mathrm{d}, J=2.0 \mathrm{~Hz})$, $6.96(1 \mathrm{H}, \mathrm{d}, J=2.0 \mathrm{~Hz}), 6.66(1 \mathrm{H}, \mathrm{d}, J=2.0 \mathrm{~Hz}), 5.82-5.77(1 \mathrm{H}, \mathrm{m}, 1 \mathrm{H})$, 5.59-5.56 $(1 \mathrm{H}, \mathrm{m}, 1 \mathrm{H}), 3.60(3 \mathrm{H}, \mathrm{s}), 3.48(3 \mathrm{H}, \mathrm{s}), 3.32\left(1 \mathrm{H}, \mathrm{dd}, J_{1,2}=17.8\right.$, $4.0 \mathrm{~Hz}), 3.15\left(1 \mathrm{H}, \mathrm{dd}, J_{1,2}=17.8,2.0 \mathrm{~Hz}\right)$.

(2R,3R)-2-(4,5-Dihydroxy-3-methoxyphenyl)-5,7-dihydroxychroman-3-yl 3,4dihydroxy-5-methoxybenzoate (38). In a similar manner to that used to prepare 2, treatment of 72 with 2-aminothiophenol gave $38(63 \%)$ as a colorless amorphous. HR-MS (FAB) calculated for $\mathrm{C}_{24} \mathrm{H}_{23} \mathrm{O}_{11} \quad[\mathrm{M}+\mathrm{H}]$ ${ }^{+} 487.1240$, found 487.1229; $[\alpha]^{\mathrm{D}}{ }_{20}=-218.1\left(c 0.04,50 \%\right.$ acetone in $\left.\mathrm{H}_{2} \mathrm{O}\right)$; IR (neat) $3285,1701,1605,1364,1221 ;{ }^{1} \mathrm{H}$ NMR $\left(500 \mathrm{MHz}, \mathrm{CD}_{3} \mathrm{OD}\right) \delta 7.10$ $(1 \mathrm{H}, \mathrm{d}, J=2.0 \mathrm{~Hz}), 7.02(1 \mathrm{H}, \mathrm{d}, J=2.0 \mathrm{~Hz}), 6.61(1 \mathrm{H}, \mathrm{d}, J=2.0 \mathrm{~Hz}), 6.58$ $(1 \mathrm{H}, \mathrm{d}, J=2.0 \mathrm{~Hz}), 5.96(2 \mathrm{H}, \mathrm{s}), 5.52(1 \mathrm{H}, \mathrm{m}), 5.23(1 \mathrm{H}, \mathrm{m}), 3.79(3 \mathrm{H}, \mathrm{s}), 3.58$ $(3 \mathrm{H}, \mathrm{s}), 3.01\left(1 \mathrm{H}, \mathrm{dd}, J_{1,2}=17.1,4.6 \mathrm{~Hz}\right), 2.86\left(1 \mathrm{H}, \mathrm{dd}, J_{1,2}=17.1,1.7 \mathrm{~Hz}\right)$; ${ }^{13} \mathrm{C}$ NMR $\left(125 \mathrm{MHz}, \mathrm{CD}_{3} \mathrm{OD}\right) \delta 165.9,156.5,156.5,148.0,147.7,144.9,144.8$, $139.2,133.4,129.3,119.8,110.6,107.3,104.9,101.7,98.1,95.2,94.4,77.5,68.9$, $55.1,54.9,25.4$.

(2R,3R)-2-(3-Methoxy-4,5-bis(2-nitrophenylsulfonyloxy)phenyl)-5,7-bis(2nitrophenylsulfonyloxy)-chroman-3-yl-3,4,5-tris(2-nitrophenylsulfonyloxy)benzoate (73). In a similar manner to that used to prepare 29, treatment of 37 with 26 gave $73(86 \%)$ as a colorless solid. HR-MS (FAB) calculated for $\mathrm{C}_{65} \mathrm{H}_{41} \mathrm{~N}_{7} \mathrm{O}_{39} \mathrm{~S}_{7} \mathrm{Na} \quad[\mathrm{M}+\mathrm{Na}]^{+} 1789.9383$, found 1789.9396; $[\alpha]^{\mathrm{D}}{ }_{20}=-37.4$ (c 0.38, acetone); IR (neat) 1709, 1618, 1593, 1545, 1442, 1391, 1364, 1308, 1196, 1116, $1006 \mathrm{~cm}^{-1}$; ${ }^{1} \mathrm{H}$ NMR $\left(270 \mathrm{MHz}\right.$, acetone- $\left.d_{6}\right) \delta 8.20-7.80(28 \mathrm{H}$, m), $7.69(2 \mathrm{H}, \mathrm{s}), 7.31(1 \mathrm{H}, \mathrm{d}, J=1.3 \mathrm{~Hz}), 7.00(1 \mathrm{H}, \mathrm{d}, J=1.3 \mathrm{~Hz}), 6.96(1 \mathrm{H}, \mathrm{d}$, $J=2.0 \mathrm{~Hz}), 6.67(1 \mathrm{H}, \mathrm{d}, J=2.0 \mathrm{~Hz}), 5.81(1 \mathrm{H}, \mathrm{m}), 5.56(1 \mathrm{H}, \mathrm{m}), 3.49(3 \mathrm{H}, \mathrm{s})$, $3.33\left(1 \mathrm{H}, \mathrm{dd}, J_{1,2}=17.8,4.0 \mathrm{~Hz}\right), 3.13\left(1 \mathrm{H}, \mathrm{dd}, J_{1,2}=17.8,2.0 \mathrm{~Hz}\right) ;{ }^{13} \mathrm{C} \mathrm{NMR}$ $\left(68 \mathrm{MHz}\right.$, acetone- $\left.d_{6}\right) \delta 162.3,156.4,154.4,149.2,149.1,148.6,143.9,143.3$, $138.8,138.1,137.9,137.7,136.9,134.1,133.8,133.7,133.4,133.0,132.8,132.5$, $132.1,130.6,130.4,129.2,128.4,128.0,127.9,127.6,126.4,126.3,126.1,125.8$, $124.7,114.4,114.0,111.2,110.8,110.5,77.4,69.0,56.9,26.8$. 
(2R,3R)-2-(4,5-Dihydroxy-3-methoxyphenyl)-5,7-dihydroxychroman-3-yl 3,4,5trihydroxybenzoate (6). In a similar manner to that used to prepare 2, treatment of 73 gave $6(63 \%)$ as a colorless amorphous. HR-MS (FAB) calculated for $\mathrm{C}_{23} \mathrm{H}_{20} \mathrm{O}_{11} \mathrm{Na}[\mathrm{M}+\mathrm{Na}]^{+} 495.0904$, found 495.0938; $[\alpha]^{\mathrm{D}}{ }_{20}=$ -148.9 (c $0.325,50 \%$ acetone in $\mathrm{H}_{2} \mathrm{O}$ ); IR (neat) $3332,1703,1618,1365$, $1244 \mathrm{~cm}^{-1} ;{ }^{1} \mathrm{H}$ NMR $\left(270 \mathrm{MHz}, \mathrm{CD}_{3} \mathrm{OD}\right) \delta 7.05(1 \mathrm{H}, \mathrm{d}, J=2.0 \mathrm{~Hz}), 7.01(1 \mathrm{H}$, d, $J=2.0 \mathrm{~Hz}), 6.51(2 \mathrm{H}, \mathrm{s}), 5.97(1 \mathrm{H}, \mathrm{d}, J=2.0 \mathrm{~Hz}), 5.95(1 \mathrm{H}, \mathrm{d}, J=2.0 \mathrm{~Hz})$, $5.49(1 \mathrm{H}, \mathrm{m}), 4.99(1 \mathrm{H}, \mathrm{m}), 3.80(3 \mathrm{H}, \mathrm{s}), 2.99\left(1 \mathrm{H}, \mathrm{dd}, J_{1,2}=17.1,4.6 \mathrm{~Hz}\right), 2.86$ $\left(1 \mathrm{H}, \mathrm{dd}, J_{1,2}=17.1,3.0 \mathrm{~Hz}\right) ;{ }^{13} \mathrm{C}$ NMR $\left(68 \mathrm{MHz}, \mathrm{CD}_{3} \mathrm{OD}\right) \delta 166.6,157.1$, $156.5,148.6,145.6,145.3,135.2,134.0,129.8,129.0,120.7,109.5,108.0,102.6$, $98.6,95.8,95.2,78.2,69.2,55.6,26.2$.

(2R,3R)-2-(3,4-Bis(2-nitrophenylsulfonyloxy)phenyl)-3-hydroxychroman-5,7-diyl bis(2-nitrophenylsulfonate) (41). In a similar manner to that used to prepare 28, treatment of (-)-Epicatechin gave $41(92 \%)$ as a colorless amorphous. HR-MS (FAB) calculated for $\mathrm{C}_{39} \mathrm{H}_{26} \mathrm{~N}_{4} \mathrm{O}_{22} \mathrm{~S}_{4}[\mathrm{M}+\mathrm{H}]^{+}$1052.9819, found 1052.9816; $[\alpha]^{\mathrm{D}}{ }_{20}=+3.83$ (c 1.06, acetone); IR (neat) 3404, 1705, 1620, $1591,1550,1440,1394,1346,1306,1182,1110,999 \mathrm{~cm}^{-1} ;{ }^{1} \mathrm{H}$ NMR $\left(500 \mathrm{MHz}\right.$, acetone- $\left.d_{6}\right) \delta 8.15-7.86(16 \mathrm{H}, \mathrm{m}), 7.57\left(1 \mathrm{H}, \mathrm{dd}, J_{1,2}=8.6\right.$, $2.0 \mathrm{~Hz}), 7.39(1 \mathrm{H}, \mathrm{d}, J=8.6 \mathrm{~Hz}), 7.38(1 \mathrm{H}, \mathrm{d}, J=2.0 \mathrm{~Hz}), 6.77(1 \mathrm{H}, \mathrm{d}$, $J=2.4 \mathrm{~Hz}), 6.51(1 \mathrm{H}, \mathrm{d}, J=2.4 \mathrm{~Hz}), 5.26(1 \mathrm{H}, \mathrm{s}), 4.33-4.26(1 \mathrm{H}, \mathrm{m}), 3.03-2.85$ $(2 \mathrm{H}, \mathrm{m}) ;{ }^{13} \mathrm{C}$ NMR $\left(126 \mathrm{MHz}\right.$, acetone- $\left.d_{6}\right) \delta 156.2,148.7,148.5,147.4,140.8$, $140.5,140.1,137.1,137.0,136.9,136.9,133.1,133.1,133.0,132.9,132.2,132.0$, $131.9,131.7,127.9,127.7,127.5,127.3,126.8,125.5,125.5,125.4,124.2,122.9$, $115.1,109.7,108.7,78.2,63.7,29.3$.

(2R,3R)-2-(3,4-Bis(2-nitrophenylsulfonyloxy)phenyl)-5,7-bis(2-nitrophenylsulfonyloxy) chroman-3-yl 3-methoxy-4,5-bis(2-nitrophenylsulfonyloxy)benzoate (74). In a similar manner to that used to prepare 29 , treatment of $\mathbf{4 1}$ with 25 gave $74(97 \%)$ as a colorless amorphous. HR-MS (FAB) calculated for $\mathrm{C}_{65} \mathrm{H}_{41} \mathrm{~N}_{7} \mathrm{O}_{39} \mathrm{~S}_{7} \mathrm{Na} \quad[\mathrm{M}+\mathrm{Na}]^{+}$1566.9831, found 1566.9817; $[\alpha]^{\mathrm{D}}{ }_{20}=-32.1$ (c 1.00, acetone); IR (neat) 1712, 1618, 1591, 1552, 1423, 1394, 1368, 1304, $1198,1117,1009 \mathrm{~cm}^{-1} ;{ }^{1} \mathrm{H}$ NMR $\left(270 \mathrm{MHz}\right.$, acetone- $\left.d_{6}\right) \delta 8.15-7.80(28 \mathrm{H}$, m), $7.62\left(1 \mathrm{H}, \mathrm{dd}, J_{1,2}=8.6,2.0 \mathrm{~Hz}\right), 7.49(1 \mathrm{H}, \mathrm{d}, J=2.0 \mathrm{~Hz}), 7.35(1 \mathrm{H}, \mathrm{d}$, $J=2.0 \mathrm{~Hz}), 7.32(1 \mathrm{H}, \mathrm{d}, J=8.6 \mathrm{~Hz}), 7.22(1 \mathrm{H}, \mathrm{d}, J=2.0 \mathrm{~Hz}), 6.98(1 \mathrm{H}, \mathrm{d}$, $J=2.0 \mathrm{~Hz}), 6.66(1 \mathrm{H}, \mathrm{d}, J=2.0 \mathrm{~Hz}), 5.74(1 \mathrm{H}, \mathrm{m}), 5.63(1 \mathrm{H}, \mathrm{s}), 3.61(3 \mathrm{H}, \mathrm{s})$, $3.33\left(1 \mathrm{H}, \mathrm{dd}, J_{1,2}=17.8,4.0 \mathrm{~Hz}\right), 3.23\left(1 \mathrm{H}, \mathrm{dd}, J_{1,2}=17.8,2.6 \mathrm{~Hz}\right) ;{ }^{13} \mathrm{C} \mathrm{NMR}$ $\left(125 \mathrm{MHz}\right.$, acetone- $\left.d_{6}\right) \delta 162.1,155.6,150.2,148.7,148.4,147.8,142.6,141.0$, $140.8,138.5,137.1,137.0,136.9,133.1,133.0,133.0,132.9,132.3,132.0,131.9$, $131.8,127.6,127.5,127.4,127.2,126.9,126.7,125.5,125.4,125.4,125.3,124.8$, $124.7,124.7,122.5,113.7,110.4,109.7,76.4,67.7,62.0,26.0$.

(2R,3R)-2-(3,4-Dihydroxyphenyl)-5,7-dihydroxychroman-3-yl 3,4-dihydroxy-5methoxybenzoate (8). In a similar manner to that used to prepare 2 , treatment of 74 gave $8(87 \%)$ as a colorless amorphous. HR-MS (FAB) calculated for $\mathrm{C}_{23} \mathrm{H}_{20} \mathrm{O}_{10} \mathrm{Na}[\mathrm{M}+\mathrm{Na}]^{+}$479.0954, found 479.0906; $[\alpha]^{\mathrm{D}}{ }_{20}=-194.4(c$ 1.10, $50 \%$ acetone in $\mathrm{H}_{2} \mathrm{O}$ ); IR (neat) 3366, 1701, 1608, 1363, $1232 \mathrm{~cm}^{-1} ;{ }^{1} \mathrm{H}$ NMR $\left(270 \mathrm{MHz}, \mathrm{CD}_{3} \mathrm{OD}\right) \delta 7.07(1 \mathrm{H}, \mathrm{d}, J=2.0 \mathrm{~Hz}), 7.02(1 \mathrm{H}, \mathrm{d}, J=2.0 \mathrm{~Hz}), 6.96$ $(1 \mathrm{H}, \mathrm{d}, J=2.0 \mathrm{~Hz}), 6.80(1 \mathrm{H}, \mathrm{dd}, J=7.9,2.0 \mathrm{~Hz}), 6.70(1 \mathrm{H}, \mathrm{d}, J=7.9 \mathrm{~Hz}), 5.98$ $(1 \mathrm{H}, \mathrm{d}, J=2.6 \mathrm{~Hz}), 5.97(1 \mathrm{H}, \mathrm{d}, J=2.6 \mathrm{~Hz}), 5.51(1 \mathrm{H}, \mathrm{m}), 5.06(1 \mathrm{H}, \mathrm{s}), 3.81$ $(3 \mathrm{H}, \mathrm{s}), 3.01(1 \mathrm{H}, \mathrm{dd}, J=17.1,4.6 \mathrm{~Hz}), 2.88(1 \mathrm{H}, \mathrm{dd}, J=17.1,2.6 \mathrm{~Hz}) ;{ }^{13} \mathrm{C}$ NMR $\left(68 \mathrm{MHz}, \mathrm{CD}_{3} \mathrm{OD}\right) \delta 167.6,157.9,157.8,157.2,149.0,146.1,146.0$, $146.0,140.6,131.5,121.5,119.2,116.0,115.1,111.9,106.2,99.3,96.6,95.8$, $78.5,70.4,56.6,26.7$.

(2R,3R)-2-(3,4-Bis(2-nitrophenylsulfonyloxy)phenyl)-5,7-bis(2-nitrophenylsulfonyloxy) chroman-3-yl 4-methoxy-3,5-bis(2-nitrophenylsulfonyloxy)benzoate (75). In a similar manner to that used to prepare 29 , treatment of 41 with 22 gave 75 (99\%) as a colorless amorphous. HR-MS (FAB) calculated for $\mathrm{C}_{65} \mathrm{H}_{41} \mathrm{~N}_{7} \mathrm{O}_{39} \mathrm{~S}_{7} \mathrm{Na} \quad[\mathrm{M}+\mathrm{Na}]^{+}$1566.9831, found 1566.9839; $[\alpha]^{\mathrm{D}}{ }_{20}=-35.4$ (c 1.00, acetone); IR (neat) 1714, 1619, 1591, 1557, 1425, 1398, 1368, 1311, $1201,1111,1007 \mathrm{~cm}^{-1} ;{ }^{1} \mathrm{H}$ NMR $\left(270 \mathrm{MHz}\right.$, acetone- $\left.d_{6}\right) \delta 8.15-7.80(28 \mathrm{H}, \mathrm{m}$, $28 \mathrm{H}), 7.59\left(1 \mathrm{H}, \mathrm{dd}, J_{1,2}=8.6,2.0 \mathrm{~Hz}\right), 7.56(2 \mathrm{H}, \mathrm{s}), 7.40(1 \mathrm{H}, \mathrm{d}, J=2.0 \mathrm{~Hz})$, $7.32(1 \mathrm{H}, \mathrm{d}, J=8.6 \mathrm{~Hz}), 6.98(1 \mathrm{H}, \mathrm{d}, J=2.0 \mathrm{~Hz}), 6.65(1 \mathrm{H}, \mathrm{d}, J=2.0 \mathrm{~Hz}), 5.71$ $(1 \mathrm{H}, \mathrm{m}), 5.59(1 \mathrm{H}, \mathrm{s}), 3.69(3 \mathrm{H}, \mathrm{s}), 3.32\left(1 \mathrm{H}, \mathrm{dd}, J_{1,2}=17.8,4.0 \mathrm{~Hz}\right), 3.10(1 \mathrm{H}$, dd, $\left.J_{1,2}=17.8,2.0 \mathrm{~Hz}\right) ;{ }^{13} \mathrm{C}$ NMR $\left(125 \mathrm{MHz}\right.$, acetone- $\left.d_{6}\right) \delta 162.1,155.6,150.2$, $148.8,148.5,147.8,142.6,141.0,140.8,138.5,137.0,136.9,133.1,133.0,132.9$, $132.9,132.3,132.0,131.9,131.8,127.6,127.5,127.4,127.1,126.9,126.7,125.5$,
$125.4,125.3,125.3,124.8,124.7,124.7,122.5,113.7,110.4,109.7,76.4,67.7$, 61.9, 26.0.

(2R,3R)-2-(3,4-Dihydroxyphenyl)-5,7-dihydroxychroman-3-yl 3,5-dihydroxy-4methoxybenzoate (9). In a similar manner to that used to prepare 2 , treatment of 75 gave $9(84 \%)$ as a colorless amorphous. $[\alpha]^{\mathrm{D}}{ }_{20}=-217.3(c 0.75,50 \%$ acetone in $\mathrm{H}_{2} \mathrm{O}$ ); IR (neat) $3367,1705,1605,1371,1240 \mathrm{~cm}^{-1}$; HR-MS (FAB) calculated for $\mathrm{C}_{23} \mathrm{H}_{20} \mathrm{O}_{10} \mathrm{Na}[\mathrm{M}+\mathrm{Na}]^{+}$479.0954, found 479.0906; ${ }^{1} \mathrm{H}$ NMR $\left(270 \mathrm{MHz}, \mathrm{CD}_{3} \mathrm{OD}\right) \delta 6.93(1 \mathrm{H}, \mathrm{d}, J=2.0 \mathrm{~Hz}), 6.92(2 \mathrm{H}, \mathrm{s}), 6.80(1 \mathrm{H}, \mathrm{dd}$, $\left.J_{1,2}=8.0,2.0 \mathrm{~Hz}\right), 6.70(1 \mathrm{H}, \mathrm{d}, J=8.0 \mathrm{~Hz}), 5.97(2 \mathrm{H}, \mathrm{s}), 5.54(1 \mathrm{H}, \mathrm{m}), 5.04$ $(1 \mathrm{H}, \mathrm{s}), 3.83(3 \mathrm{H}, \mathrm{s}), 3.01\left(1 \mathrm{H}, \mathrm{dd}, J_{1,2}=17.8,4.6 \mathrm{~Hz}\right), 2.85\left(1 \mathrm{H}, \mathrm{dd}, J_{1,2}=17.8\right.$, $2.6 \mathrm{~Hz}) ;{ }^{13} \mathrm{C}$ NMR $\left(68 \mathrm{MHz}, \mathrm{CD}_{3} \mathrm{OD}\right) \delta 167.1,157.9,157.8,157.2,151.5$, $146.0,141.1,131.4,126.5,119.3,116.0,115.0,110.3,99.3,96.6,95.9,78.5,70.3$, $60.7,26.8$.

5-((2S,3R)-3-Hydroxy-5,7-bis(2-nitrophenylsulfonyloxy)chroman-2-yl)benzene1,2,3-triyl tris(2-nitrobenzenesulfonate) (42). In a similar manner to that used to prepare 28, treatment of (-)-gallocatechin gave $\mathbf{4 2}(94 \%)$ as a colorless amorphous. HR-MS (FAB) calculated for $\mathrm{C}_{45} \mathrm{H}_{30} \mathrm{~N}_{5} \mathrm{O}_{27} \mathrm{~S}_{5}[\mathrm{M}+\mathrm{H}]^{+}$1231.9732, found 1231.9717; $[\alpha]_{20}^{\mathrm{D}}=-1.61$ (c 1.00, acetone); IR (neat) 3406, 1701, 1618, $1589,1542,1421,1388,1362,1305,1222,1193,1114,1008 \mathrm{~cm}^{-1} ;{ }^{1} \mathrm{H}$ NMR $\left(500 \mathrm{MHz}\right.$, acetone- $\left.d_{6}\right) \delta 8.15-7.85(20 \mathrm{H}, \mathrm{m}), 7.32(2 \mathrm{H}, \mathrm{s}), 6.74(1 \mathrm{H}, \mathrm{d}$, $J=2.6 \mathrm{~Hz}), 6.55(1 \mathrm{H}, \mathrm{d}, J=2.6 \mathrm{~Hz}), 5.00(1 \mathrm{H}, \mathrm{d}, J=7.3 \mathrm{~Hz}), 4.75(1 \mathrm{H}, \mathrm{d}$, $J=5.1 \mathrm{~Hz}), 3.90-3.97(1 \mathrm{H}, \mathrm{m}), 2.82\left(1 \mathrm{H}, \mathrm{dd}, J_{1,2}=16.8,5.4 \mathrm{~Hz}\right), 2.66(1 \mathrm{H}, \mathrm{dd}$, $\left.J_{1,2}=16.8,8.6 \mathrm{~Hz}\right) ;{ }^{13} \mathrm{C}$ NMR $\left(125 \mathrm{MHz}\right.$, acetone- $\left.d_{6}\right) \delta 155.2,148.7,148.5$, $148.2,148.0,147.6,142.8,140.2,137.2,137.2,137.1,136.9,134.6,133.2,133.2$, $133.1,132.9,132.2,132.1,131.9,131.6,128.6,127.3,127.1,126.7,125.5,121.5$, $115.7,109.7,109.3,80.2,65.6,27.8$

5-(2S,3R)-5,7-Bis(2-nitrophenylsulfonyloxy)-2-(3,4,5-tris(2-nitrophenylsulfonyloxy) phenyl) chroman-3-yl-3-methoxy-4,5-bis(2-nitrophenylsulfonyloxy)benzoate (76). In a similar manner to that used to prepare 29 , treatment of $\mathbf{4 2}$ with $\mathbf{2 5}$ gave $76(93 \%)$ as a colorless amorphous. HR-MS (FAB) calculated for $\mathrm{C}_{65} \mathrm{H}_{41} \mathrm{~N}_{7} \mathrm{O}_{39} \mathrm{~S}_{7} \mathrm{Na} \quad[\mathrm{M}+\mathrm{Na}]^{+} 1789.9383$, found $1789.9375 ;[\alpha]^{\mathrm{D}}{ }_{20}=+4.03$ (c 1.25, acetone); IR (neat) 1717, 1618, 1591, 1550, 1419, 1391, 1362, 1305, $1188,1105,1000 \mathrm{~cm}^{-1} ;{ }^{1} \mathrm{H}$ NMR $\left(500 \mathrm{MHz}\right.$, acetone- $\left.d_{6}\right) \delta 8.20-7.75(28 \mathrm{H}$, m), $7.56(1 \mathrm{H}, \mathrm{d}, J=2.0 \mathrm{~Hz}), 7.39(2 \mathrm{H}, \mathrm{s}), 7.33(1 \mathrm{H}, \mathrm{d}, J=2.0 \mathrm{~Hz}), 6.87(1 \mathrm{H}, \mathrm{d}$, $J=2.0 \mathrm{~Hz}), 6.66(1 \mathrm{H}, \mathrm{d}, J=2.0 \mathrm{~Hz}), 5.63(1 \mathrm{H}, \mathrm{d}, J=5.8 \mathrm{~Hz}), 5.44-5.40(1 \mathrm{H}$, m), $3.65(3 \mathrm{H}, \mathrm{s}), 3.06\left(1 \mathrm{H}, \mathrm{dd}, J_{1,2}=17.8,6.3 \mathrm{~Hz}\right), 2.81\left(1 \mathrm{H}, \mathrm{dd}, J_{1,2}=17.8\right.$, $4.6 \mathrm{~Hz}) ;{ }^{13} \mathrm{C}$ NMR $\left(125 \mathrm{MHz}\right.$, acetone- $\left.d_{6}\right) \delta 162.7,154.5,153.7,148.7,148.4$, 148.2, 148.2, 148.1, 148.0, 143.2, 142.6, 138.8, 137.4, 137.3, 137.2, 137.0, 136.4, $133.2,133.2,133.0,132.9,132.2,132.1,132.0,131.9,131.7,131.4,129.6,129.4$, $128.2,127.66,127.1,126.8,126.6,125.6,125.5,125.4,125.2,121.4,116.4$, $113.8,113.0,110.0,109.9,76.9,68.7,56.4,23.1$.

(2S,3R)-5,7-Dihydroxy-2-(3,4,5-trihydroxyphenyl)chroman-3-yl 3,4-dihydroxy-5methoxybenzoate (10). In a similar manner to that used to prepare 2, treatment of $\mathbf{7 6}$ gave $\mathbf{1 0}(81 \%)$ as a colorless amorphous. HR-MS (FAB) calculated for $\mathrm{C}_{23} \mathrm{H}_{20} \mathrm{O}_{11} \mathrm{Na}[\mathrm{M}+\mathrm{Na}]^{+}$495.0904, found 495.0873; [ $\left.\alpha\right]$ $\mathrm{D}_{20}=+13.9\left(c 0.30,50 \%\right.$ acetone in $\left.\mathrm{H}_{2} \mathrm{O}\right)$; IR (neat) $3369,1701,1611,1375$, $1240 \mathrm{~cm}^{-1} ;{ }^{1} \mathrm{H}$ NMR $\left(270 \mathrm{MHz}, \mathrm{CD}_{3} \mathrm{OD}\right) \delta 7.07(1 \mathrm{H}, \mathrm{d}, J=2.0 \mathrm{~Hz}), 7.01(1 \mathrm{H}$, d, $J=2.0 \mathrm{~Hz}), 6.43(2 \mathrm{H}, \mathrm{s}), 5.96(1 \mathrm{H}, \mathrm{d}, J=2.0 \mathrm{~Hz}), 5.94(1 \mathrm{H}, \mathrm{d}, J=2.0 \mathrm{~Hz})$, $5.35-5.25(1 \mathrm{H}, \mathrm{m}), 4.99(1 \mathrm{H}, \mathrm{d}, J=6.6 \mathrm{~Hz}), 3.81(3 \mathrm{H}, \mathrm{s}), 2.90(1 \mathrm{H}, \mathrm{dd}$, $\left.J_{1,2}=16.5,4.6 \mathrm{~Hz}\right), 2.70\left(1 \mathrm{H}, \mathrm{dd}, J_{1,2}=16.5,6.0 \mathrm{~Hz}\right) ;{ }^{13} \mathrm{C} \mathrm{NMR}(68 \mathrm{MHz}$, $\left.\mathrm{CD}_{3} \mathrm{OD}\right) \delta 167.6,158.1,157.7,156.6,149.1,147.0,146.2,140.7,134.1,130.9$, $121.5,111.9,106.6,106.2,99.7,96.5,95.6,79.7,71.7,56.7,24.7$.

5-(2S,3R)-5,7-Bis(2-nitrophenylsulfonyloxy)-2-(3,4,5-tris(2-nitrophenylsulfonyloxy) phenyl) chroman-3-yl-4-methoxy-3,5-bis(2-nitrophenylsulfonyloxy)benzoate (77). In a similar manner to that used to prepare 29 , treatment of $\mathbf{4 2}$ with $\mathbf{2 2}$ gave $\mathbf{7 7}$ as a crude colorless amorphous. The crude $\mathbf{7 7}$ was used in the next step without further purification. ${ }^{1} \mathrm{H}$ NMR $\left(500 \mathrm{MHz}\right.$, acetone- $\left.d_{6}\right) \delta 8.20-7.80(28 \mathrm{H}, \mathrm{m})$, $7.68(2 \mathrm{H}, \mathrm{s}), 7.33(2 \mathrm{H}, \mathrm{s}), 6.86(1 \mathrm{H}, \mathrm{d}, J=2.3 \mathrm{~Hz}), 6.64(1 \mathrm{H}, \mathrm{d}, J=2.3 \mathrm{~Hz}), 5.63$ $(1 \mathrm{H}, \mathrm{d}, J=5.8 \mathrm{~Hz}), 5.37-5.42(1 \mathrm{H}, \mathrm{m}), 3.67(3 \mathrm{H}, \mathrm{s}), 3.01\left(1 \mathrm{H}, \mathrm{dd}, J_{1,2}=18.4\right.$, $5.2 \mathrm{~Hz}), 2.71\left(1 \mathrm{H}, \mathrm{dd}, J_{1,2}=18.4,4.6 \mathrm{~Hz}\right)$.

(2S,3R)-5,7-Dihydroxy-2-(3,4,5-trihydroxyphenyl)chroman-3-yl 3,5-dihydroxy-4methoxybenzoate (11). In a similar manner to that used to prepare 2 , 
treatment of crude 77 gave 11 (71\%, two steps) as a colorless amorphous. HR-MS (FAB) calculated for $\mathrm{C}_{23} \mathrm{H}_{20} \mathrm{O}_{11} \mathrm{Na}[\mathrm{M}+\mathrm{Na}]^{+}$495.0904, found 495.0885; $[\alpha]^{\mathrm{D}}{ }_{20}=+13.7\left(c \quad 0.25,50 \%\right.$ acetone in $\left.\mathrm{H}_{2} \mathrm{O}\right)$; IR (neat) 3300, 1701, 1604, 1377, $1238 \mathrm{~cm}^{-1}$; ${ }^{1} \mathrm{H}$ NMR $\left(270 \mathrm{MHz}, \mathrm{CD}_{3} \mathrm{OD}\right) \delta 6.95(2 \mathrm{H}, \mathrm{s})$, $6.41(2 \mathrm{H}, \mathrm{s}), 5.96(2 \mathrm{H}, \mathrm{s}), 5.42-5.35(1 \mathrm{H}, \mathrm{m}), 5.04(1 \mathrm{H}, \mathrm{d}, J=5.9 \mathrm{~Hz}), 3.84$ $(3 \mathrm{H}, \mathrm{s}), 2.80\left(1 \mathrm{H}, \mathrm{dd}, J_{1,2}=16.5,5.3 \mathrm{~Hz}\right), 2.71\left(1 \mathrm{H}, \mathrm{dd}, J_{1,2}=16.5,5.3 \mathrm{~Hz}\right) ;{ }^{13} \mathrm{C}$ NMR $\left(68 \mathrm{MHz}, \mathrm{CD}_{3} \mathrm{OD}\right) \delta 167.1,158.1,158.0,157.6,157.5,156.3,151.6$, $147.0,141.3,134.0,130.9,126.5,110.2,106.4,99.5,79.2,71.4,60.7,23.9$.

(2R,3S)-2-(3,4-Bis(2-nitrophenylsulfonyloxy)phenyl)-3-hydroxychroman-5,7-diyl bis(2-nitrophenylsulfonate) (43). In a manner similar to that used to prepare 28, treatment of (+)-catechin gave $\mathbf{4 3}(94 \%)$ as a colorless amorphous. HR-MS (FAB) calculated for $\mathrm{C}_{39} \mathrm{H}_{26} \mathrm{~N}_{4} \mathrm{O}_{22} \mathrm{~S}_{4}[\mathrm{M}+\mathrm{H}]^{+}$1052.9819, found 1052.9843; $[\alpha]_{20}^{\mathrm{D}}=+8.54$ (c 1.00, acetone); IR (neat) $3498,1713,1618,1595,1545,1419$, $1392,1350,1304,1191,1112,1015 \mathrm{~cm}^{-1} ;{ }^{1} \mathrm{H}$ NMR $\left(270 \mathrm{MHz}, \mathrm{CD}_{3} \mathrm{CN}\right) \delta$ 8.07-8.01 (4H, m), 7.91-7.71 (12H, m), 7.33-7.23 (3H, m), $6.74(1 \mathrm{H}, \mathrm{d}$, $J=2.5 \mathrm{~Hz}), 6.57(1 \mathrm{H}, \mathrm{d}, J=2.5 \mathrm{~Hz}), 4.83(1 \mathrm{H}, \mathrm{d}, J=7.3 \mathrm{~Hz}), 3.98(1 \mathrm{H}, \mathrm{m})$, $2.98\left(1 \mathrm{H}, \mathrm{dd}, J_{1,2}=17.0,5.0 \mathrm{~Hz}\right), 2.80\left(1 \mathrm{H}, \mathrm{dd}, J_{1,2}=17.0,8.0 \mathrm{H}\right) ;{ }^{13} \mathrm{C} \mathrm{NMR}$ $\left(68 \mathrm{MHz}, \mathrm{CD}_{3} \mathrm{CN}\right) \delta 156.1,149.3,149.1,148.9,148.5,141.8,141.6,140.7$, $137.8,137.7,133.9,133.8,133.7,133.1,132.8,132.7,132.6,128.4,128.2,128.0$, $127.4,126.2,126.2,125.4,123.2,116.3,110.6,110.1,81.1,66.1,27.7$.

(2R,3S)-2-(3,4-Bis(2-nitrophenylsulfonyloxy)phenyl)-5,7-bis(2-nitrophenylsulfonyloxy) chroman-3-yl 3-methoxy-4,5-bis(2-nitrophenylsulfonyloxy)benzoate (78). In a similar manner to that used to prepare 29 , treatment of 43 with 25 gave $78(74 \%)$ as a colorless amorphous. HR-MS (FAB) calculated for $\mathrm{C}_{65} \mathrm{H}_{41} \mathrm{~N}_{7} \mathrm{O}_{39} \mathrm{~S}_{7} \mathrm{Na} \quad[\mathrm{M}+\mathrm{Na}]^{+}$1566.9831, found 1566.9819; $[\alpha]^{\mathrm{D}}{ }_{20}=+22.4$ (c 1.07, acetone); IR (neat) 1718, 1618, 1591, 1554, 1417, 1385, 1356, 1304, $1184,1119,1000 \mathrm{~cm}^{-1}$; ${ }^{1} \mathrm{H}$ NMR $\left(500 \mathrm{MHz}\right.$, acetone- $\left.d_{6}\right) \delta 8.17-7.85(28 \mathrm{H}$, m), $7.54(1 \mathrm{H}, \mathrm{d}, J=2.3 \mathrm{~Hz}), 7.49(1 \mathrm{H}, \mathrm{dd}, J=8.6,2.3 \mathrm{~Hz}), 7.39(1 \mathrm{H}, \mathrm{d}$, $J=8.6 \mathrm{~Hz}), 7.32(1 \mathrm{H}, \mathrm{d}, J=2.3 \mathrm{~Hz}), 7.31(1 \mathrm{H}, \mathrm{d}, J=2.3 \mathrm{~Hz}), 6.89(1 \mathrm{H}, \mathrm{d}$, $J=2.0 \mathrm{~Hz}), 6.65(1 \mathrm{H}, \mathrm{d}, J=2.0 \mathrm{~Hz}), 5.59(1 \mathrm{H}, \mathrm{d}, J=5.8 \mathrm{~Hz}), 5.46-5.42(1 \mathrm{H}$, m), $3.65(3 \mathrm{H}, \mathrm{s}), 3.04\left(1 \mathrm{H}, \mathrm{dd}, J_{1,2}=17.2,5.2 \mathrm{~Hz}\right), 2.77\left(1 \mathrm{H}, \mathrm{dd}, J_{1,2}=17.2\right.$, $4.6 \mathrm{~Hz}) ;{ }^{13} \mathrm{C}$ NMR $\left(125 \mathrm{MHz}\right.$, acetone- $\left.d_{6}\right) \delta 162.7,154.8,153.8,148.7,148.5$, $148.4,148.2,148.1,148.0,142.6,141.3,141.1,138.6,137.2,137.2,137.1,137.0$, $136.4,135.2,133.2,133.1,133.0,133.0,132.9,132.3,131.9,131.9,131.8,131.4$, $129.6,129.5,127.6,127.5,127.3,127.1,126.9,126.6,125.6,125.5,125.5,125.4$, $125.4,125.2,125.1,122.3,116.3,113.9,112.9,110.1,109.8,77.1,68.9$, $56.4,23.2$.

(2R,3S)-2-(3,4-Dihydroxyphenyl)-5,7-dihydroxychroman-3-yl 3,4-dihydroxy-5methoxybenzoate (12). In a similar manner to that used to prepare 2, treatment of $\mathbf{7 8}$ gave $12(99 \%)$ as a colorless amorphous. HR-MS (FAB) calculated for $\mathrm{C}_{23} \mathrm{H}_{20} \mathrm{O}_{10} \mathrm{Na} \quad[\mathrm{M}+\mathrm{Na}]^{+}$479.0954, found 479.0994, $[\alpha]^{\mathrm{D}_{20}}=+142.5\left(c 0.34,50 \%\right.$ acetone in $\left.\mathrm{H}_{2} \mathrm{O}\right)$; IR (neat) 3331, 1705, 1612, $1385,1231 \mathrm{~cm}^{-1} ;{ }^{1} \mathrm{H}$ NMR $\left(270 \mathrm{MHz}, \mathrm{CD}_{3} \mathrm{OD}\right) \delta 7.07(1 \mathrm{H}, \mathrm{d}, J=2.0 \mathrm{~Hz})$, $7.01(1 \mathrm{H}, \mathrm{d}, J=2.0 \mathrm{~Hz}), 6.86(1 \mathrm{H}, \mathrm{d}, J=2.0 \mathrm{~Hz}), 6.71-6.76(2 \mathrm{H}, \mathrm{m}), 5.97(1 \mathrm{H}$, d, $J=2.0 \mathrm{~Hz}), 5.94(1 \mathrm{H}, \mathrm{d}, J=2.0 \mathrm{~Hz}), 5.28-5.37(1 \mathrm{H}, \mathrm{m}), 5.03(1 \mathrm{H}, \mathrm{d}$, $J=6.6 \mathrm{~Hz}), 3.82(3 \mathrm{H}, \mathrm{s}), 2.92\left(1 \mathrm{H}, \mathrm{dd}, J_{1,2}=16.5,5.3 \mathrm{~Hz}\right), 2.71(1 \mathrm{H}, \mathrm{dd}$, $\left.J_{1,2}=16.5,7.3 \mathrm{~Hz}\right) ;{ }^{13} \mathrm{C} \mathrm{NMR}\left(68 \mathrm{MHz}, \mathrm{CD}_{3} \mathrm{OD}\right) \delta 167.4,158.2,157.7,156.7$, $149.0,146.4,146.2,140.7,131.4,121.4,119.4,116.2,114.7,111.9,106.2,99.8$, $96.5,95.6,79.7,71.2,56.7,25.1$.

(2R,3S)-2-(3,4-Bis(2-nitrophenylsulfonyloxy)phenyl)-5,7-bis(2-nitrophenylsulfonyloxy) chroman-3-yl 4-methoxy-3,5-bis(2-nitrophenylsulfonyloxy)benzoate (79). In a similar manner to that used to prepare 29, treatment of 43 with 22 gave $79(89 \%)$ as a colorless amorphous. HR-MS (FAB) calculated for $\mathrm{C}_{65} \mathrm{H}_{41} \mathrm{~N}_{7} \mathrm{O}_{39} \mathrm{~S}_{7} \mathrm{Na} \quad[\mathrm{M}+\mathrm{Na}]^{+}$1566.9831, found 1566.9825; $[\alpha]^{\mathrm{D}}{ }_{20}=+21.0$ (c 1.10, acetone); IR (neat) 1705, 1618, 1593, 1554, 1418, 1395, 1365, 1315, $1177,1119,1011 \mathrm{~cm}^{-1}$; ${ }^{1} \mathrm{H}$ NMR $\left(500 \mathrm{MHz}\right.$, acetone- $\left.d_{6}\right) \delta 8.18-7.88(28 \mathrm{H}$, m), $7.67(2 \mathrm{H}, \mathrm{s}), 7.45(1 \mathrm{H}, \mathrm{dd}, J=8.6,2.3 \mathrm{~Hz}), 7.39(1 \mathrm{H}, \mathrm{d}, J=8.6 \mathrm{~Hz}), 7.26$ $(1 \mathrm{H}, \mathrm{d}, J=2.3 \mathrm{~Hz}), 6.88(1 \mathrm{H}, \mathrm{d}, J=2.0 \mathrm{~Hz}), 6.65(1 \mathrm{H}, \mathrm{d}, J=2.0 \mathrm{~Hz}), 5.58$ $(1 \mathrm{H}, \mathrm{d}, J=5.2 \mathrm{~Hz}), 5.43-5.39(1 \mathrm{H}, \mathrm{m}), 3.69(3 \mathrm{H}, \mathrm{s}), 3.00\left(1 \mathrm{H}, \mathrm{dd}, J_{1,2}=17.8\right.$, $5.8 \mathrm{~Hz}), 2.66\left(1 \mathrm{H}, \mathrm{dd}, J_{1,2}=17.8,4.6 \mathrm{~Hz}\right) ;{ }^{13} \mathrm{C}$ NMR $\left(125 \mathrm{MHz}\right.$, acetone- $\left.d_{6}\right) \delta$ $162.2,154.7,150.3,148.8,148.5,148.5,148.4,148.1,148.1,142.6,141.3,141.1$, $138.5,137.2,137.2,137.1,137.1,137.0,133.1,133.0,132.9,132.3,132.0,131.9$, $131.8,131.8,127.6,127.5,127.3,127.0,126.9,126.6,125.6,125.5,125.4,125.1$, $124.8,122.1,113.8,110.1,109.9,77.0,68.6,61.9,22.9$.
(2R,3S)-2-(3,4-Dihydroxyphenyl)-5,7-dihydroxychroman-3-yl 3,5-dihydroxy-4methoxybenzoate (13). In a similar manner to that used to prepare 2, treatment of $\mathbf{7 9}$ gave $\mathbf{1 3}(89 \%)$ as a colorless amorphous. HR-MS (FAB) calculated for $\mathrm{C}_{23} \mathrm{H}_{20} \mathrm{O}_{10} \mathrm{Na} \quad[\mathrm{M}+\mathrm{Na}]^{+}$479.0954, found 479.0994; $[\alpha]^{\mathrm{D}}{ }_{20}=+110.6\left(c 0.80,50 \%\right.$ acetone in $\left.\mathrm{H}_{2} \mathrm{O}\right)$; IR (neat) 3367, 1701, 1604, $1367,1234 \mathrm{~cm}^{-1}$; ${ }^{1} \mathrm{H}$ NMR $\left(270 \mathrm{MHz}, \mathrm{CD}_{3} \mathrm{OD}\right) \delta 6.94(2 \mathrm{H}, \mathrm{s}), 6.83(1 \mathrm{H}, \mathrm{s})$, $6.72(2 \mathrm{H}, \mathrm{s}), 5.97-5.93(2 \mathrm{H}, \mathrm{m}), 5.41-5.34(1 \mathrm{H}, \mathrm{m}), 5.05(1 \mathrm{H}, \mathrm{d}, J=5.9 \mathrm{~Hz})$, $3.84(3 \mathrm{H}, \mathrm{s}), 2.85\left(1 \mathrm{H}, \mathrm{dd}, J_{1,2}=16.5,5.3 \mathrm{~Hz}\right), 2.71\left(1 \mathrm{H}, \mathrm{dd}, J_{1,2}=16.5,6.6 \mathrm{~Hz}\right)$; ${ }^{13} \mathrm{C}$ NMR $\left(68 \mathrm{MHz}, \mathrm{CD}_{3} \mathrm{OD}\right) \delta 167.1,158.2,157.6,156.5,151.6,146.3,146.3$, $131.3,126.5,119.3,116.2,114.4,110.2,99.6,96.5,95.6,79.3,71.5,60.7,24.5$.

5,7-Di-O-(2-nitrobenzenesulfonyl)catechin (51). To a solution of boric acid $(100 \mathrm{~g}, 1.6 \mathrm{~mol})$ and $\mathrm{NaOH}(20 \mathrm{~g})$ in $\mathrm{H}_{2} \mathrm{O}(1300 \mathrm{ml})$ was added 49 (10.0 g, $34.5 \mathrm{mmol}$ ) and the resulting solution was adjusted to $\mathrm{pH} 9.0$ by the addition of $1 \mathrm{~m} \mathrm{NaOH}$. Then, 2-nitrobenzenesulfonyl chloride (15.3 g, $69.0 \mathrm{mmol})$ in toluene $(220 \mathrm{ml})$ was added dropwise over $30 \mathrm{~min}$ and the mixture was stirred at room temperature for $7 \mathrm{~h}$. The reaction was quenched with $1 \mathrm{M} \mathrm{HCl}$ and extracted with ethyl acetate. The organic phase was dried over anhydrous sodium sulfate and evaporated under reduced pressure. The residue was purified by silica gel flash column chromatography $\left(1-3 \% \mathrm{MeOH}\right.$ in $\mathrm{CH}_{2} \mathrm{Cl}_{2}$ ) to afford $51(15.9 \mathrm{~g}, 70 \%)$ as a pale yellow amorphous solid. HR-MS (ESI) calculated for $\mathrm{C}_{27} \mathrm{H}_{20} \mathrm{~N}_{2} \mathrm{O}_{14} \mathrm{~S}_{2} \mathrm{Na} \quad[\mathrm{M}+\mathrm{Na}]^{+}$683.0248, found 683.0281; $[\alpha]^{20}{ }_{D}=+13.3$ (c 1.0, acetone) IR (neat) 3398, 1701, 1618, 1591, 1545, 1439, $1389,1366,1196,1110,1032,997,780 \mathrm{~cm}^{-1} ;{ }^{1} \mathrm{H}$ NMR $\left(500 \mathrm{MHz}\right.$, acetone- $\left.d_{6}\right)$ $\delta 8.09-8.00(3 \mathrm{H}, \mathrm{m}), 7.98-7.75(5 \mathrm{H}, \mathrm{m}), 6.78(1 \mathrm{H}, \mathrm{d}, J=8.0 \mathrm{~Hz}), 6.73(1 \mathrm{H}, \mathrm{dd}$, $\left.J_{1,2}=6.5,2.0 \mathrm{~Hz}\right), 6.60\left(1 \mathrm{H}, \mathrm{dd}, J_{1,2}=8.0,2.0 \mathrm{~Hz}\right), 6.55(1 \mathrm{H}, \mathrm{d}, J=2.0 \mathrm{~Hz}), 4.85$ $(1 \mathrm{H}, \mathrm{d}, J=6.0 \mathrm{~Hz}), 4.39(1 \mathrm{H}, \mathrm{d}, J=4.0 \mathrm{~Hz}), 4.10-4.05(1 \mathrm{H}, \mathrm{m}), 2.66(1 \mathrm{H}, \mathrm{dd}$, $\left.J_{1,2}=17.0,7.5 \mathrm{~Hz}\right), 2.53\left(1 \mathrm{H}, \mathrm{dd}, J_{1,2}=17.0,5.0 \mathrm{~Hz}\right) ;{ }^{13} \mathrm{C} \mathrm{NMR}(126 \mathrm{MHz}$, acetone- $\left.d_{6}\right) \delta 157.1,149.5,149.2,149.0,148.5,148.4,145.9,145.8,137.8,137.7$, $133.8,133.6,133.0,132.5,130.8,128.2,127.6,126.2,126.2,119.1,116.5,116.0$, $114.5,110.3,109.3,82.7,66.2,27.6$.

5,7-Di-O-(2-nitrobenzenesulfonyl)epicatechin (52). In a similar manner to that used to prepare $\mathbf{5 1}$, treatment of $\mathbf{5 0}(5.0 \mathrm{~g})$ gave $\mathbf{5 2}(7.1 \mathrm{~g}, 63 \%)$ as a pale yellow amorphous solid. HR-MS (ESI) calculated for $\mathrm{C}_{27} \mathrm{H}_{20} \mathrm{~N}_{2} \mathrm{O}_{14} \mathrm{~S}_{2} \mathrm{Na}[\mathrm{M}+\mathrm{Na}]^{+}$ 683.0248, found 683.0255; $[\alpha]^{20}{ }_{D}=+6.5$ (c 1.00, acetone); IR (neat) 3421, 1691, $1617,1385,1195,1110,997,781 \mathrm{~cm}^{-1} ;{ }^{1} \mathrm{H}$ NMR $\left(500 \mathrm{MHz}\right.$, acetone- $\left.d_{6}\right) \delta$ 8.17-8.08 (4H, m, 4H), 8.06-8.01 (2H, m, 2H), 8.00-7.91 (2H, m), $7.91(1 \mathrm{H}$, br s), $7.88(1 \mathrm{H}$, br s), $7.00(1 \mathrm{H}$, br s), $6.79(2 \mathrm{H}, \mathrm{br} \mathrm{s}), 6.77(1 \mathrm{H}, \mathrm{d}, J=2.0 \mathrm{~Hz})$, $6.47(1 \mathrm{H}, \mathrm{d}, J=2.0 \mathrm{~Hz}), 5.02(1 \mathrm{H}, \mathrm{br} \mathrm{s}), 4.30-4.20(1 \mathrm{H}, \mathrm{m}), 4.06(1 \mathrm{H}, \mathrm{d}$, $J=4.5 \mathrm{~Hz}), 3.00-2.85(2 \mathrm{H}, \mathrm{m}) ;{ }^{13} \mathrm{C}$ NMR $\left(126 \mathrm{MHz}\right.$, acetone- $\left.d_{6}\right) \delta 157.0$, $148.7,148.5,147.5,144.8,144.7,136.9,133.1,132.9,132.2,131.8,130.0,127.6$, $126.9,125.5,125.4,118.5,115.3,114.8,114.4,109.6,108.2,79.5,64.3,29.1$.

5,7-Di-O-(2-nitrobenzenesulfonyl)epigallocatechin (53). In a similar manner to that used to prepare $\mathbf{5 1}$, treatment of $\mathbf{2 7}(4.0 \mathrm{~g})$ gave $\mathbf{5 3}(4.6 \mathrm{~g}, 52 \%)$ as a brown amorphous solid. HR-MS (ESI) calculated for $\mathrm{C}_{27} \mathrm{H}_{20} \mathrm{~N}_{2} \mathrm{O}_{15} \mathrm{~S}_{2} \mathrm{Na}[\mathrm{M}+\mathrm{Na}]^{+}$ 699.0197, found 699.0191; $[\alpha]^{20}{ }_{\mathrm{D}}=+4.1$ (c 1.00, acetone); IR (neat) 3342, 1701, $1620,1591,1547,1441,1385,1368,1194,1111,997,781 \mathrm{~cm}^{-1} ;{ }^{1} \mathrm{H}$ NMR $\left(500 \mathrm{MHz}\right.$, acetone- $\left.d_{6}\right) \delta 8.20-7.75(8 \mathrm{H}, \mathrm{m}), 7.34(1 \mathrm{H}, \mathrm{s}), 7.00(1 \mathrm{H}, \mathrm{br} \mathrm{s})$, $6.75(1 \mathrm{H}, \mathrm{d}, J=2.5 \mathrm{~Hz}), 6.51(2 \mathrm{H}, \mathrm{s}), 6.47(1 \mathrm{H}, \mathrm{d}, J=2.0 \mathrm{~Hz}), 4.96(1 \mathrm{H}, \mathrm{br} \mathrm{s})$, $4.26-4.20(1 \mathrm{H}, \mathrm{m}), 4.01(1 \mathrm{H}, \mathrm{d}, J=4.5 \mathrm{~Hz}), 2.93\left(1 \mathrm{H}, \mathrm{dd}, J_{1,2}=16.5,4.0 \mathrm{~Hz}\right)$, $2.53\left(1 \mathrm{H}, \mathrm{dd}, J_{1,2}=16.5,4.0 \mathrm{~Hz}\right) ;{ }^{13} \mathrm{C}$ NMR $\left(126 \mathrm{MHz}\right.$, acetone- $\left.d_{6}\right) \delta 157.7$, $149.4,148.2,146.1,137.7,133.8,133.6,133.2,133.0,132.6,130.0,128.3,127.6$, $126.2,126.2,110.3,108.9,106.7,80.1,65.1,30.0$.

(2R,3R)-5,7,5,7-Tetra-O-(2-nitrobenzenesulfonyl)neotheaflavin (56). To a solution of $50(1.0 \mathrm{~g}, 1.5 \mathrm{mmol})$ in $\mathrm{MeCN}(15 \mathrm{ml})$ was added $\mathrm{Pb}(\mathrm{OAc})_{4}(806 \mathrm{mg}$, $4.5 \mathrm{mmol}$ ) at $0{ }^{\circ} \mathrm{C}$. The resulting suspension was stirred for $10 \mathrm{~min}$ at $0{ }^{\circ} \mathrm{C}$. The reaction mixture was added to benzene, and then the mixture was filtered through a pad of celite. Then, the filtrate was evaporated under reduced pressure, and the resulting crude product was used in the next reaction without further purification.

The crude product 55 was dissolved in $\mathrm{MeCN} / \mathrm{CH}_{2} \mathrm{Cl}_{2}(1: 4,25 \mathrm{ml})$. To this solution were added MS3A (1.0 g) and Ns-epigallocatchin (53) $(342 \mathrm{mg}$, $505 \mu \mathrm{mol})$ in $\mathrm{MeCN} / \mathrm{CH}_{2} \mathrm{Cl}_{2}(1: 4,10 \mathrm{ml})$ at $0{ }^{\circ} \mathrm{C}$. The resulting suspension was stirred for $20 \mathrm{~min}$ at $0^{\circ} \mathrm{C}$. After the addition of $\mathrm{H}_{2} \mathrm{O}$, the mixture was stirred for $5 \mathrm{~min}$ at room temperature. The reaction mixture was filtered, and 
then the filtrate was extracted with AcOEt, the organic phase was washed with $\mathrm{H}_{2} \mathrm{O}$, and evaporated under reduced pressure. The residue was purified by silica gel flash column chromatography $\left(\mathrm{CH}_{2} \mathrm{Cl}_{2}: \mathrm{MeOH}=98: 2\right)$ to afford 56 (327 mg, 50\%) as an orange amorphous solid. HR-MS (ESI) calculated for $\mathrm{C}_{53} \mathrm{H}_{36} \mathrm{~N}_{4} \mathrm{O}_{28} \mathrm{~S}_{4} \mathrm{Na} \quad[\mathrm{M}+\mathrm{Na}]^{+}$1327.0291, found 1327.0284; $[\alpha]^{20}{ }_{\mathrm{D}}=-6.6$ (c 0.20, acetone); IR (neat) 3352, 1701, 1618, 1591, 1541, 1477, 1437, 1384, $1361,1232,1190,1106,1058,1030,995850,775,736 \mathrm{~cm}^{-1} ;{ }^{1} \mathrm{H}$ NMR $\left(500 \mathrm{MHz}\right.$, acetone- $\left.d_{6}\right) \delta 8.18-7.86(16 \mathrm{H}, \mathrm{m}), 7.51(1 \mathrm{H}, \mathrm{s}), 7.47(1 \mathrm{H}, \mathrm{s}), 6.88$ $(1 \mathrm{H}, \mathrm{s}), 6.83(1 \mathrm{H}, \mathrm{br} \mathrm{s}), 6.63(1 \mathrm{H}, \mathrm{d}, J=2.0 \mathrm{~Hz}), 6.53(1 \mathrm{H}, \mathrm{d}, J=2.0 \mathrm{~Hz}), 5.85$ $(1 \mathrm{H}, \mathrm{br} s), 5.17(1 \mathrm{H}, \mathrm{s}), 4.49(1 \mathrm{H}, \mathrm{s}), 4.31-2.98(1 \mathrm{H}, \mathrm{m}), 3.10(1 \mathrm{H}, \mathrm{dd}$, $\left.J_{1,2}=17.5,2.5 \mathrm{~Hz}\right), 3.05\left(1 \mathrm{H}, \mathrm{dd}, J_{1,2}=17.5,2.5 \mathrm{~Hz}\right), 2.80\left(1 \mathrm{H}, \mathrm{dd}, J_{1,2}=17.0\right.$, $7.5 \mathrm{~Hz}), 2.65-2.50(1 \mathrm{H}, \mathrm{br} \mathrm{s}) ;{ }^{13} \mathrm{C}$ NMR $\left(125 \mathrm{MHz}\right.$, acetone- $\left.d_{6}\right) \delta 184.8,156.9$, $156.7,154.5,151.2,149.2,149.2,149.1,149.0,148.8,148.4,148.1,146.3,137.6$, $137.6,137.5,133.8,133.7,133.7,133.6132 .9,132.8,132.5,132.3,130.4,129.7$, $128.1,128.0,127.9,127.5,127.3,126.2,126.1,126.1,126.1,121.6,118.6,116.3$, $115.7,110.5,110.3,109.7,109.4,82.0,69.7,66.5,64.7,55.2,29.6,29.5$.

Neotheaflavin (5). To a suspension of $\mathrm{Cs}_{2} \mathrm{CO}_{3}(223 \mathrm{mg}, 0.17 \mathrm{mmol})$ and thiophenol $(0.17 \mathrm{ml}, 1.7 \mathrm{mmol})$ in $\operatorname{MeCN} / \operatorname{DMF}(1: 2,2.7 \mathrm{ml})$ was added the solution of 56 in $\mathrm{MeCN}(3.0 \mathrm{ml})$ at $0{ }^{\circ} \mathrm{C}$ and the reaction mixture was stirred at the same temperature for $2.0 \mathrm{~h}$. The reaction was quenched with $1 \mathrm{M} \mathrm{HCl}$ aqueous and extracted with AcOEt. The organic phase was evaporated under reduced pressure. The residue was purified by preparative TLC $\left(\mathrm{CH}_{2} \mathrm{Cl}_{2}\right.$ : $\mathrm{MeOH}=9: 1)$ to afford $5(53.1 \mathrm{mg}, 55 \%)$ as a orange amorphous solid. HR-MS (ESI) calculated for $\mathrm{C}_{29} \mathrm{H}_{24} \mathrm{O}_{12} \mathrm{Na}[\mathrm{M}+\mathrm{Na}]^{+}$587.1159, found 587.1130; $[\alpha]^{20}{ }_{D}=-122.1$ (c 0.20, acetone); IR (neat) 3362, 1699, 1622, 1607, 1506, $1472,1429,1361,1236,1193,1143,1099,1076,1046,1012,823 \mathrm{~cm}^{-1} ;{ }^{1} \mathrm{H}$ NMR $\left(500 \mathrm{MHz}\right.$, acetone- $\left.d_{6}\right) \delta 8.80(1 \mathrm{H}, \mathrm{br} \mathrm{s}), 8.26(1 \mathrm{H}, \mathrm{s}), 7.77(1 \mathrm{H}, \mathrm{s}), 7.66$ $(1 \mathrm{H}, \mathrm{s}), 6.06(1 \mathrm{H}, \mathrm{s}), 6.03(1 \mathrm{H}, \mathrm{s}), 5.95(1 \mathrm{H}, \mathrm{s}), 5.94(1 \mathrm{H}, \mathrm{s}), 5.60(1 \mathrm{H}, \mathrm{d}$, $J=5.0 \mathrm{~Hz}), 5.01(1 \mathrm{H}, \mathrm{s}), 4.36(1 \mathrm{H}, \mathrm{br} \mathrm{s}), 4.12-4.06(1 \mathrm{H}, \mathrm{m}), 3.00-2.75(3 \mathrm{H}$, m), $2.63\left(1 \mathrm{H}, \mathrm{dd}, J_{1,2}=16.0,9.0 \mathrm{~Hz}\right) ;{ }^{13} \mathrm{C}$ NMR $\left(125 \mathrm{MHz}\right.$, acetone- $\left.d_{6}\right) \delta 184.9$, 157.7, 157.5, 157.4, 157.1, 156.7, 156.6, 154.4, 150.6, 146.3, 134.9, 132.3, 130.9, $128.7,122.4,121.7,119.3,100.8,99.3,96.496 .3,95.6,95.4,81.7,79.3,69.5$, 66.7, 30.6, 29.4. Analytical data for neotheaflavin (5) in ref. 16.

(2R,3R)-5,7,5,7-Tetra-O-(2-nitrobenzenesulfonyl)theaflavin (64). To a solution of $52(500 \mathrm{mg}, 757 \mathrm{mmol})$ in $\mathrm{MeCN}(7 \mathrm{ml})$ was added $\mathrm{Pb}(\mathrm{OAc})_{4}(268 \mathrm{mg}$, $605 \mathrm{mmol}$ ) at $0{ }^{\circ} \mathrm{C}$. The resulting suspension was stirred for $10 \mathrm{~min}$ at the same temparature. The reaction mixture was added benzene $(20 \mathrm{ml})$, and then the mixture was filtered with celite pad. Then the filtrate was evaporated under reduced pressure, and the resulting crude product was used in the next reaction without further purification.

The crude product 63 was dissolved in $\mathrm{MeCN} / \mathrm{CH}_{2} \mathrm{Cl}_{2}(1: 4,12 \mathrm{ml})$. The solution was added MS3A (300 mg), Ns-epigallocatchin 53 (171 mg, $252 \mathrm{mmol})$ in $\mathrm{MeCN} / \mathrm{CH}_{2} \mathrm{Cl}_{2}(1: 4,4 \mathrm{ml})$ at $0{ }^{\circ} \mathrm{C}$. The resulting suspension was stirred for $20 \mathrm{~min}$ at $0{ }^{\circ} \mathrm{C}$. After the addition of $\mathrm{H}_{2} \mathrm{O}$, the mixture was stirred for $5 \mathrm{~min}$ at room temperature. The reaction mixture was filtered, and then the filtrate was extracted with AcOEt, the organic phase was washed with $\mathrm{H}_{2} \mathrm{O}$, and evaporated under reduced pressure. The residue was purified by chromatography on silica gel column $\left(\mathrm{CH}_{2} \mathrm{Cl}_{2}: \mathrm{MeOH}=9: 1\right)$ to afford mixture of 64 and $\mathbf{5 2}$ as an orange amorphous solid, and the mixture of product was used in the next reaction without further purification. HR-MS (ESI) calculated for $\mathrm{C}_{53} \mathrm{H}_{36} \mathrm{~N}_{4} \mathrm{O}_{28} \mathrm{~S}_{4} \mathrm{Na}[\mathrm{M}+\mathrm{Na}]^{+}$1327.0291, found 1327.0245.

Theaflavine (4). To a solution of the crude $64(249 \mathrm{mg})$ in MeCN/DMF (1:2, $3.0 \mathrm{ml})$ were added $\mathrm{Cs}_{2} \mathrm{CO}_{3}(257 \mathrm{mg}, 789 \mathrm{mmol})$ and thiophenol $39(0.09 \mathrm{ml}$, $879 \mathrm{mmol})$ at $0{ }^{\circ} \mathrm{C}$ and the reaction mixture was stirred for $3 \mathrm{~h}$ at the same temperature. The reaction was quenched with $1 \mathrm{~N} \mathrm{HCl}$ aqueous and extracted with AcOEt. The organic layer was evaporated under reduced pressure and the residue was purified by HPLC to afford Theaflavin (4) $(16 \mathrm{mg}, 11 \%$ for two steps) as an orange amorphous solid. HR-MS (ESI) calculated for $\mathrm{C}_{29} \mathrm{H}_{24} \mathrm{O}_{12} \mathrm{Na}$ $[\mathrm{M}+\mathrm{Na}]^{+} 587.1159$, found 587.1164; $[\alpha]^{20}{ }_{\mathrm{D}}=-274.8$ (c 0.20 , acetone); IR (neat) $3275,1691,1624,1600,1507,1460,1419,1352,1230,1197,1138,1089$, $1060,1041,1010,891,805,706 \mathrm{~cm}^{-1} ;{ }^{1} \mathrm{H}$ NMR $\left(500 \mathrm{MHz}, \mathrm{CD}_{3} \mathrm{OD}\right) \delta 7.94$ $(1 \mathrm{H}, \mathrm{s}), 7.81(1 \mathrm{H}, \mathrm{s}), 7.33(1 \mathrm{H}, \mathrm{s}), 6.00(1 \mathrm{H}, \mathrm{d}, J=2.0 \mathrm{~Hz}), 5.97(1 \mathrm{H}, \mathrm{d}$, $J=2.0 \mathrm{~Hz}), 5.95(1 \mathrm{H}, \mathrm{d}, J=2.0 \mathrm{~Hz}), 5.94(1 \mathrm{H}, \mathrm{d}, J=2.0 \mathrm{~Hz}), 5.62(1 \mathrm{H}, \mathrm{s}), 4.86$ $(1 \mathrm{H}, \mathrm{s}), 4.47-4.41(1 \mathrm{H}, \mathrm{m}), 4.32-4.25(1 \mathrm{H}, \mathrm{m}), 2.96\left(1 \mathrm{H}, \mathrm{dd}, J_{1,2}=17.0\right.$,
$4.0 \mathrm{~Hz}), 2.93\left(1 \mathrm{H}, \mathrm{dd}, J_{1,2}=17.0,4.0 \mathrm{~Hz}\right), 2.82(1 \mathrm{H}$, br d, $J=16.0 \mathrm{~Hz}), 2.80$ $(1 \mathrm{H}$, br d, $J=16.0 \mathrm{~Hz}) ;{ }^{13} \mathrm{C}$ NMR $\left(126 \mathrm{MHz}, \mathrm{CD}_{3} \mathrm{OD}\right) \delta 184.7,157.0,156.9$, $156.6,156.4,156.1,156.1,155.4,149.8,145.2,133.4,130.2,127.7,125.0,122.7$, 120.9, 117.1, 99.0, 98.4, 95.5, 94.8, 94.3, 80.0, 75.9, 65.3, 64.4, 28.6, 28.1. Analytical data for theaflavin (4) in ref. 16.

(2R,3S)-3-Hydroxy-2-(3,4,6-trihydroxy-5-oxo-5H-benzo[7]annulen-1-yl)chroman5,7-diyl bis(2-nitrobenzenesulfonate) (80). To a solution of 51 (100 mg, $152 \mu \mathrm{mol})$ in $\mathrm{MeCN}(2 \mathrm{ml})$ was added $\mathrm{Pb}(\mathrm{OAc})_{4}(53.7 \mathrm{mg}, 121 \mu \mathrm{mol})$ at $0{ }^{\circ} \mathrm{C}$. The resulting suspension was stirred for $10 \mathrm{~min}$ at $0{ }^{\circ} \mathrm{C}$. To the reaction mixture was added benzene $(6.7 \mathrm{ml})$, and then the mixture was filtered through a pad of celite. Then, the filtrate was evaporated under reduced pressure, and the resulting crude product was used in the next reaction without further purification.

The crude product 55 was dissolved in $\mathrm{MeCN} / \mathrm{CH}_{2} \mathrm{Cl}_{2}$ (1:4, $\left.1.65 \mathrm{ml}\right)$. To this solution were added MS3A $(30 \mathrm{mg})$ and pyrogallol $65(19 \mathrm{mg}, 150 \mu \mathrm{mol})$ in $\mathrm{MeCN} / \mathrm{CH}_{2} \mathrm{Cl}_{2}(1: 4,0.50 \mathrm{ml})$ at $0{ }^{\circ} \mathrm{C}$. The resulting suspension was stirred for 20 min at $0{ }^{\circ} \mathrm{C}$. After the addition of $\mathrm{H}_{2} \mathrm{O}$, the mixture was stirred for $5 \mathrm{~min}$ at room temperature. The reaction mixture was filtered, and then the filtrate was extracted with AcOEt, the organic phase was washed with $\mathrm{H}_{2} \mathrm{O}$ and evaporated under reduced pressure. The residue was purified by preparative TLC $\left(\mathrm{CH}_{2} \mathrm{Cl}_{2}\right.$ : $\mathrm{MeOH}=98: 2)$ to afford $80(19.5 \mathrm{mg})$ as a orange amorphous solid. HR-MS (ESI) calculated for $\mathrm{C}_{32} \mathrm{H}_{22} \mathrm{~N}_{2} \mathrm{O}_{16} \mathrm{~S}_{2} \mathrm{Na}[\mathrm{M}+\mathrm{Na}]^{+} 777.0320$, found 777.0269; $[\alpha]^{20}{ }_{\mathrm{D}}=+52.9$ (c 0.20, acetone); IR (neat) $3420,1554,1384,1195,1111$, $997 \mathrm{~cm}^{-1}$; ${ }^{1} \mathrm{H}$ NMR $\left(500 \mathrm{MHz}\right.$, methanol- $\left.d_{4}\right) \delta 8.15-7.90(8 \mathrm{H}, \mathrm{m}), 7.88(1 \mathrm{H}$, d, $J=12.0 \mathrm{~Hz}), 7.47(1 \mathrm{H}, \mathrm{s}), 7.25(1 \mathrm{H}, \mathrm{d}, J=9.2 \mathrm{~Hz}), 6.90\left(1 \mathrm{H}, \mathrm{dd}, J_{1,2}=12.0\right.$, $9.2 \mathrm{~Hz}), 6.79(1 \mathrm{H}, \mathrm{d}, J=2.3 \mathrm{~Hz}), 6.60(1 \mathrm{H}, \mathrm{d}, J=2.3 \mathrm{~Hz}), 5.75(1 \mathrm{H}, \mathrm{d}$, $J=6.2 \mathrm{~Hz}), 4.31-4.24(1 \mathrm{H}, \mathrm{m}), 2.82\left(1 \mathrm{H}, \mathrm{dd}, J_{1,2}=16.9,7.7 \mathrm{~Hz}\right), 2.65(1 \mathrm{H}, \mathrm{dd}$, $\left.J_{1,2}=16.9,4.3 \mathrm{~Hz}\right) ;{ }^{13} \mathrm{C}$ NMR $\left(126 \mathrm{MHz}\right.$, acetone- $\left.d_{6}\right) \delta 157.0,148.9,148.4$, $145.8,145.8,137.7,137.6,133.7,133.6,133.0,132.5,130.8,128.1,127.5,126.2$, $126.1,119.1,116.5,116.0,114.4,110.3,109.3,82.6,66.1,27.5$.

3,4,6-Trihydroxy-1-((2R,3S)-3,5,7-trihydroxychroman-2-yl)-5H-benzo[7]annulen5-one (67). To a suspension of $\mathrm{Cs}_{2} \mathrm{CO}_{3}(40.0 \mathrm{mg}, 53.0 \mu \mathrm{mol})$ and thiophenol $(55 \mu \mathrm{l}, 0.53 \mathrm{mmol})$ in $\mathrm{MeCN} / \mathrm{DMF}(1: 2,0.9 \mathrm{ml})$ was added solution of the crude of 80 in $\mathrm{MeCN}(1.0 \mathrm{ml})$ at $0{ }^{\circ} \mathrm{C}$ and the reaction mixture was stirred at the same temperature for $2 \mathrm{~h}$. The reaction was quenched with $1 \mathrm{M} \mathrm{HCl}$ aqueous and extracted with AcOEt. The organic phase was evaporated under reduced pressure. The residue was purified by preparative TLC $\left(\mathrm{CH}_{2} \mathrm{Cl}_{2}\right.$ : $\mathrm{MeOH}=9: 1)$ to afford $67(15.9 \mathrm{mg}, 78 \%)$ as a orange amorphous solid. HR-MS (ESI) calculated for $\mathrm{C}_{20} \mathrm{H}_{16} \mathrm{O}_{8} \mathrm{Na}[\mathrm{M}+\mathrm{Na}]^{+}$407.0737, found 407.0748; $[\alpha]^{20}{ }_{\mathrm{D}}=-24.8$ (c 0.20, acetone); IR (neat) $3319,1602,1411,1327,1253,1070$, $825 \mathrm{~cm}^{-1}$; ${ }^{1} \mathrm{H}$ NMR $\left(500 \mathrm{MHz}\right.$, methanol- $\left.d_{4}\right) \delta 7.84(1 \mathrm{H}, \mathrm{d}, J=12.5 \mathrm{~Hz}), 7.60$ $(1 \mathrm{H}, \mathrm{s}), 7.15(1 \mathrm{H}, \mathrm{d}, J=9.1 \mathrm{~Hz}), 6.80\left(1 \mathrm{H}, \mathrm{dd}, J_{1,2}=12.5,9.1 \mathrm{~Hz}\right), 6.79(1 \mathrm{H}, \mathrm{d}$, $J=2.3 \mathrm{~Hz}), 6.60(1 \mathrm{H}, \mathrm{d}, J=2.3 \mathrm{~Hz}), 5.75(1 \mathrm{H}, \mathrm{d}, J=6.2 \mathrm{~Hz}), 4.20-4.13(1 \mathrm{H}$, m), $2.86\left(1 \mathrm{H}, \mathrm{dd}, J_{1,2}=16.3,5.3 \mathrm{~Hz}\right), 2.62\left(1 \mathrm{H}, \mathrm{dd}, J_{1,2}=16.1,8.5 \mathrm{~Hz}\right) ;{ }^{13} \mathrm{C}$ NMR $\left(126 \mathrm{MHz}\right.$, methanol- $\left.d_{4}\right) \delta 186.3,158.0,157.7,156.7,156.6,151.9,147.1$, 131.5, 130.0, 124.1, 122.9, 122.6, 118.6, 100.7, 96.7, 95.5, 80.2, 68.9, 49.7, 28.9.

Methyl 3,4,6-trihydroxy-1-((2R,3S)-3-hydroxy-5,7-bis(((2-nitrophenyl)sulfonyl) oxy) chroman-2-yl)-5-oxo-5H-benzo[7]annulene-8-carboxylate (81). To a solution of $51(200 \mathrm{mg}, 303 \mu \mathrm{mol})$ in $\mathrm{MeCN}(3 \mathrm{ml})$ was added $\mathrm{Pb}(\mathrm{OAc})_{4}(161 \mathrm{mg}$, $363 \mu \mathrm{mol})$ at $0{ }^{\circ} \mathrm{C}$. The resulting suspension was stirred for $10 \mathrm{~min}$ at $0{ }^{\circ} \mathrm{C}$. The reaction mixture was added to benzene, and then the mixture was filtered through a pad of celite. Then, the filtrate was evaporated under reduced pressure, and the resulting crude product was used in the next reaction without further purification.

The crude product 55 was dissolved in $\mathrm{MeCN} / \mathrm{CH}_{2} \mathrm{Cl}_{2}(1: 4,5.0 \mathrm{ml})$. To the solution were added MS3A (200 mg) and methyl gallate (66) (18.6 mg, $101 \mu \mathrm{mol})$ in $\mathrm{MeCN} / \mathrm{CH}_{2} \mathrm{Cl}_{2}(1: 4,2.0 \mathrm{ml})$ at $0{ }^{\circ} \mathrm{C}$. The resulting suspension was stirred for $20 \mathrm{~min}$ at $0^{\circ} \mathrm{C}$. After the addition of $\mathrm{H}_{2} \mathrm{O}$, the mixture was stirred for $5 \mathrm{~min}$ at room temperature. The reaction mixture was filtered, and then the filtrate was extracted with AcOEt, the organic phase was washed with $\mathrm{H}_{2} \mathrm{O}$ and evaporated under reduced pressure. The residue was purified by preparative TLC $\left(\mathrm{CH}_{2} \mathrm{Cl}_{2}: \mathrm{MeOH}=98: 2\right)$ to afford $81(40.6 \mathrm{mg}, 50 \%)$ as a orange amorphous solid. HR-MS (ESI) calculated for $\mathrm{C}_{34} \mathrm{H}_{24} \mathrm{~N}_{2} \mathrm{NaO}_{18} \mathrm{~S}_{2}$ $[\mathrm{M}+\mathrm{Na}]^{+} 835.0358$, found 835.0343; $[\alpha]^{20}{ }_{\mathrm{D}}=+9.6$ (c 0.20, acetone); IR (neat) 
$3369,1701,1385,1248,1195,1111,999,781 \mathrm{~cm}^{-1} ;{ }^{1} \mathrm{H}$ NMR $(500 \mathrm{MHz}$, acetone- $\left.d_{6}\right) \delta 8.88(1 \mathrm{H}, \mathrm{s}), 8.12-7.93(8 \mathrm{H}, \mathrm{m}), 7.76(1 \mathrm{H}, \mathrm{s}), 7.58(1 \mathrm{H}, \mathrm{s}), 6.82$ $(1 \mathrm{H}, \mathrm{d}, J=2.3 \mathrm{~Hz}), 6.61(1 \mathrm{H}, \mathrm{d}, J=2.3 \mathrm{~Hz}), 5.62(1 \mathrm{H}, \mathrm{d}, J=7.5 \mathrm{~Hz}), 4.33-4.26$ $(1 \mathrm{H}, \mathrm{m}), 3.84(1 \mathrm{H}, \mathrm{s}), 2.90(1 \mathrm{H}, \mathrm{m}), 2.74\left(1 \mathrm{H}, \mathrm{dd}, J_{1,2}=17.2,8.6 \mathrm{~Hz}\right) ;{ }^{13} \mathrm{C}$ NMR $\left(126 \mathrm{MHz}\right.$, acetone- $\left.d_{6}\right) \delta 186.8,168.5,157.6,155.4,153.1,150.3,150.1,149.9$, $149.7,149.4,138.7,134.7,134.5,134.3,133.9,133.6,133.5,129.1,129.0$, $128.3,127.2,127.1,125.1,123.6,123.1,117.5,116.8,111.4,110.8,82.1,67.5$, $54.3,31.4$.

Methyl 3,4,6-trihydroxy-5-oxo-1-((2R,3S)-3,5,7-trihydroxychroman-2-yl)-5Hbenzo[7] annulene-8-carboxylate (68). To a suspension of $\mathrm{Cs}_{2} \mathrm{CO}_{3}(52.1 \mathrm{mg}$, $64.1 \mu \mathrm{mol})$ and thiophenol $(66 \mu \mathrm{l}, 641 \mu \mathrm{mol})$ in $\mathrm{MeCN} / \mathrm{DMF}(1: 2,0.9 \mathrm{~m}$;) was added solution of $81 \mathrm{in} \mathrm{MeCN}(1.0 \mathrm{ml})$ at $0{ }^{\circ} \mathrm{C}$ and the reaction mixture was stirred at the same temperature for $30 \mathrm{~min}$. The reaction was quenched with $1 \mathrm{M} \mathrm{HCl}$ aqueous and extracted with AcOEt. The organic phase was evaporated under reduced pressure. The residue was purified by preparative TLC $\left(\mathrm{CH}_{2} \mathrm{Cl}_{2}\right.$ : $\mathrm{MeOH}=9: 1)$ to afford $68(13.4 \mathrm{mg}, 47 \%)$ as a orange amorphous solid. HR-MS (ESI) calculated for $\mathrm{C}_{22} \mathrm{H}_{18} \mathrm{O}_{10} \mathrm{Na}[\mathrm{M}+\mathrm{Na}]^{+}$465.0792, found 465.0795; $[\alpha]^{20}{ }_{D}=-44.6$ (c 0.20, acetone); IR (neat) $3346,1701,1608,1225 \mathrm{~cm}^{-1} ;{ }^{1} \mathrm{H}$ NMR $\left(500 \mathrm{MHz}\right.$, methanol- $\left.d_{4}\right) \delta 8.92(1 \mathrm{H}, \mathrm{s}), 7.69(1 \mathrm{H}, \mathrm{s}), 7.62(1 \mathrm{H}, \mathrm{s}), 5.97$ $(1 \mathrm{H}, \mathrm{d}, J=1.7 \mathrm{~Hz}), 5.91(1 \mathrm{H}, \mathrm{d}, J=1.7 \mathrm{~Hz}), 5.34(1 \mathrm{H}, \mathrm{d}, J=8.0 \mathrm{~Hz}), 4.35-4.13$ $(1 \mathrm{H}, \mathrm{m}), 3.82(1 \mathrm{H}, \mathrm{s}), 2.97\left(1 \mathrm{H}, \mathrm{dd}, J_{1,2}=16.1,5.7 \mathrm{~Hz}\right), 2.61(1 \mathrm{H}, \mathrm{dd}$, $\left.J_{1,2}=16.0,9.2 \mathrm{~Hz}\right) ;{ }^{13} \mathrm{C}$ NMR $\left(126 \mathrm{MHz}\right.$, methanol- $\left.d_{4}\right) \delta 186.8,181.9,158.1$, $157.7,156.6,155.0,152.4,149.7,134.6,134.4,128.7,124.0,123.5,112.8,115.9$, $100.8,96.7,95.5,81.3,69.1,53.5,29.7$.

\section{CONFLICT OF INTEREST}

The authors declare no conflict of interest.

\section{ACKNOWLEDGEMENTS}

We thank Dr Masayuki Suzuki (Mitsui Norin Co., Ltd) for providing samples of (-)-EGC, (-)-EC and (-)-GC. This work was financially supported by a grant from the Shizuoka Prefecture and Shizuoka City Collaboration of Regional Entities for the Advancement of Technological Excellence, a grant from the Japan Science and Technology Agency (JST), the Uehara Memorial Foundation (Y.H.), MEXT/JSPS KAKENHI grant numbers 23390007 and 24790017, Grants-in-Aid for Scientific Research on Priority Areas 12045232 and 24105530 from the Ministry of Education, Culture, Sports, Science and Technology (MEXT) of Japan, and a grant for Platform for Drug Discovery, Informatics, and Structural Life Science from the Ministry of Education, Culture, Sports, Science and Technology.

1 Fukuyama, T., Jow, C.-K. \& Cheung, M. 2- and 4-Nitrobenzenesulfonamides: exceptionally versatile means for preparation of secondary amines and protection of amines. Tetrahedron Lett. 36, 6373-6374 (1995).

2 Nagle, D. G., Ferreira, D. \& Zhou, Y. D. Epigallocatechin-3-gallate (EGCG): chemical and biomedical perspectives. Phytochemistry 67, 1849-1855 (2006).

3 Higdon, J. V. \& Frei, B. Tea catechins and polyphenols: health effects, metabolism, and antioxidant functions. Crit. Rev. Food Sci. Nutr. 43, 89-143 (2003).

4 Zaveri, N. T. Green tea and its polyphenolic catechins: medicinal uses in cancer and noncancer applications. Life Sci. 78, 2073-2080 (2006).

5 Maeda-Yamamoto, M. et al. Association of suppression of extracellular signal-regulated kinase phosphorylation by epigallocatechin gallate with the reduction of matrix metalloproteinase activities in human fibrosarcoma HT1080 cells. J. Agric. Food Chem. 51, 1858-1863 (2003).

6 Oku, N. et al. Inhibitory effect of green tea polyphenols on membrane-type 1 matrix metalloproteinase, MT1-MMP. Biol. Pharm. Bull. 26, 1235-1238 (2003).

7 Maeda-Yamamoto, M., Ema, K. \& Shibuichi, I. In vitro and in vivo anti-allergic effects of 'benifuuki' green tea containing $O$-methylated catechin and ginger extract enhancement. Cytotechnology 55, 135-142 (2007).

8 Maeda-Yamamoto, M. et al. O-methylated catechins from tea leaves inhibit multiple protein kinases in mast cells. J. Immunol. 172, 4486-4492 (2004).
9 Fujimura, Y. et al. Antiallergic tea catechin, (-)-epigallocatechin-3-0-(3-0-methyl)gallate, suppresses FcepsilonRI expression in human basophilic KU812 cells. J. Agric. Food Chem. 50, 5729-5734 (2002).

10 Suzuki, M., Yoshino, K., Maeda-Yamamoto, M., Miyase, T. \& Sano, M. Inhibitory effects of tea catechins and $O$-methylated derivatives of (-)-epigallocatechin-3-O-gallate on mouse type IV allergy. J. Agric. Food Chem. 48, 5649-5653 (2000).

11 Tachibana, H. et al. Identification of a methylated tea catechin as an inhibitor of degranulation in human basophilic KU812 cells. Biosci. Biotechnol. Biochem. 64, 452-454 (2000).

12 Miyase, T. \& Sano, M. Jpn. Kokai \& Tokkyo Koho JP253879 (2001).

13 Tanaka, H., Miyoshi, H., Chuang, Y.-C., Ando, Y. \& Takahashi, T. Solid-phase synthesis of epigallocatechin gallate derivatives. Angew. Chem. Int. Ed. Engl. 46, 5934-5937 (2007).

14 Furuta, T. et al. Concise synthesis of dideoxy-epigallocatechin gallate (DO-EGCG) and evaluation of its anti-influenza virus activity. Bioorg. Med. Chem. Lett. 17, 3095-3098 (2007).

15 Hirooka, Y., Nitta, M., Furuta, T. \& Kan, T. Efficient synthesis of optically active gallocatechin- 3-gallate derivatives via 6-endo cyclization. Synlett 3234-3238 (2008).

16 EGC has been readily obtained from EGCg by enzymatic hydrolysis mediated by tannase, see, Battestin, V., Macedo, G. A. \& De Freitas, V. A. P. Hydrolysis of epigallocatechin gallate using a tannase from Paecilomyces variotii. Food Chem. 108, 228-233 (2008).

17 Kan, T. \& Fukuyama, T. Highly versatile synthesis of nitrogen-containing compounds by means of nitrobenzenesulfonamides. J. Syn. Org. Chem. Jpn 59, 779-789 (2001).

18 Kan, T. \& Fukuyama, T. Ns strategies: a highly versatile synthetic method for amines. Chem. Commun. (Comb) 353-359 (2003).

19 Koyama, Y., Yamaguchi, R. \& Suzuki, K. Total synthesis and structure assignment of the anthrone C-glycoside cassialoin. Angew. Chem., Int. Ed. Engl. 47, 1084-1087 (2008).

20 Chang, J., Chen, R., Guo, R., Dong, C. \& Zhao, K. Synthesis, separation, and theoretical studies of chiral biphenyl lignans ( $\alpha$ - and $\beta$-DDB). Helv. Chem. Acta 86, 2239-2246 (2003).

21 Honda, M., Morita, H. \& Nagakura, I. Deprotection of allyl groups with sulfinic acids and palladium catalyst. J. Org. Chem. 62, 8932-8936 (1997).

22 Aihara, Y. et al. Regioselective synthesis of methylated epigallocatechin gallate via nitrobenzenesulfonyl (Ns) protecting group. Bioorg. Med. Chem. Lett. 19, 4171-4174 (2009)

23 Van Dyk, M. S., Steynberg, J. P., Steynberg, P. J. \& Ferreira, D. Selective O-methylation of polyhydroxyflavan-3-ols via benzyl carbonates. Tetrahedron Lett. 31, 2643-2646 (1990)

24 Matoba, M., Kajimoto, T. \& Node, M. Application of odorless thiols for the cleavage of 2- and 4-nitrobenzenesulfonamides. Synth. Commun. 38, 1194-1200 (2008).

25 Roberts, E. A. H. Oxidation-reduction potentials in tea fermentation. Chem. Ind 1354-1355 (1957).

26 Takino, Y., Ferretti, A., Flanagan, V., Gianturco, M. \& Vogel, M. Structure of theaflavin, a polyphenol of black tea. Tetrahedron Lett. 6, 4019-4025 (1965).

27 Friedman, M, Henika, P. R., Levin, C. E., Mandrell, R. E. \& Kozuku, N. Antimicrobial activities of tea catechins and theaflavins and tea extracts against Bacillus cereus. J. Food Prot. 69, 354-361 (2006).

28 Lorenz, M. et al. Green and black tea are equally potent stimuli of NO production and vasodilation: new insights into tea ingredients involved. Basic Res. Cardiol. 104, 100-110 (2009).

29 Wu, Y.-Y., Li, W., Xu, Y., Jin, E-H. \& Tu, Y.-Y. Evaluation of the antioxidant effects of four main theaflavin derivatives through chemiluminescence and DNA damage analyses. J. Zhejiang Univ. Sci. B 12, 744-751 (2011).

$30 \mathrm{Oka}, \mathrm{Y}$. et al. Tea polyphenols inhibit rat osteoclast formation and differentiation. J. Pharmacol. Sci. 118, 55-64 (2012).

31 Oyama, K., Yoshida, K. \& Kondo, T. Recent progress in the synthesis of flavonoids: from monomers to supra-complex molecules. Curr. Org. Chem. 15, 2567-2607 (2011).

32 Tanaka, T., Mine, C., Inoue, K., Matsuda, M. \& Kouno, I. Synthesis of theaflavin from epicatechin and epigallocatechin by plant homogenates and role of epicatechin quinone in the synthesis and degradation of theaflavin. J. Agric. Food Chem. 50, 2142-2148 (2002).

33 Sang, S. et al. Enzymatic synthesis of tea theaflavin derivatives and their anti-inflammatory and cytotoxic activities. Bioorg. Med. Chem. 12, 459-467 (2004).

34 Tu, Y. Y., Xu, X. Q., Xia, H. L. \& Watanabe, N. Optimization of theaflavin biosynthesis from tea polyphenols using an immobilized enzyme system and response surface methodology. Biotechnol. Lett. 27, 269-274 (2005).

35 Yanase, E., Sawaki, K. \& Nakatsuka, S. The isolation of a bicyclo[3.2.1] intermediate during formation of benzotropolones, a common nucleus found in black tea pigments: theaflavins. Synlett 2661-2663 (2005).

36 Kawabe, Y. et al. Synthesis of theaflavin via biomimetic oxidative coupling reaction. Synlett 479-482 (2013). 